In ac losses in type // superconductors three contributing factors may be distinguished.

These are connected with the three parameters in the Critical State Model: the critical

current density $J_{c}$, surface screening currents denoted by $B_{\mathrm{en}}\left(B_{\mathrm{a}}\right)$ and $B_{\mathrm{ex}}\left(B_{\mathrm{a}}\right)$, and

the flux conductivity $\sigma_{\mathrm{ff} .}$ In this paper the authors present an inductive technique

which makes it possible to determine these three parameters separately. For this purpose,

ac magnetic fields of trapzoidal waveform are applied. The theoretical background of

the technique will be discussed together with the experimental equipment designed for

these experiments. The technique has been verified by means of numerical model

calculations. Finally, some experimental results are given to illustrate the possibilities

of the technique.

\title{
Trapezoidal ac magnetic fields: a powerful technique for the investigation of type 11 superconductors
}

\author{
E.A. Gijsbertse, M. Caspari and L.J.M. van de Klundert
}

Since the early sixties, the dc behaviour of hard superconductors has been described in terms of the Critical State Model (CSM). In this model, superconducting bulk currents and surface currents are distinguished. Under ac conditions, over-critical currents due to flux flow effects have also to be taken into account to explain the ac losses. In the literature, many experimental methods have been developed to determine one or two of the parameters of the CSM. It is the aim of this contribution to present an experimental technique with which it is possible to determine the three parameters separately.

In the original formulation of the CSM by Bean ${ }^{1}$ and London ${ }^{2}$, the only parameter is the critical current density $j_{c}$ which describes the supercurrents throughout the bulk of the superconductor. In a more generalized $\mathrm{CSM}^{3,4}$ surface currents which prevent flux entry and flux exit at the surface and flux flow effects are also included. A summary of this generalized CSM is given in the next section. Many techniques have been developed to study the properties of type II superconductors; excellent reviews have been given by Campbell and Evetts ${ }^{5}$ and Ullmaier. ${ }^{6}$

A very prominent role in the determination of the various parameters of the CSM is assigned to the inductive techniques; a discussion of the essentials of these techniques will be given later with the emphasis on non-sinusoidal ac field waveform. Following this, the inductive response of a superconducting slab to an ac magnetic field of trapezoidal waveform will be discussed theoretically. It will be demonstrated that the flux distribution inside the sample, and therefore the current distribution, can be determined experimentally with this technique. Apart from this, the technique can separate the various contributions (bulk currents, surface currents and flux flow) to the ac losses, as will be shown. The method has been verified with the help of numerical model calculations. Finally, a summary of the various possibilities of the use of trapezoidal ac fields will be given.

Experiments have been performed in a large range of $\mathrm{d} B_{\mathrm{a}} / \mathrm{d} t$ values and amplitudes. A detailed discussion of the equipment used in these experiments is given. The central point

The authors are from the Twente University of Technology, Department of Applied Physics, PO Box 217, 7500 AE Enschede, The Netherlands. Paper received 11 July 1980. in this equipment is the waveform generator (trapezium generator) which not only generates the trapezoidal waveform, but also controls the data acquisition process. Some experimental results obtained in this way are shown. A first discussion of some of these results is already given in a previous publication; ${ }^{3}$ more results and a detailed discussion will be published separately.

\section{The Critical State Model}

Prior to a discussion of the experimental technique, the formulation of the Critical State Model (CSM) and its extensions, as it will be used throughout this paper, will be outlined. In the framework of the CSM, a local average magnetic induction $B$ and a local average field intensity $H$ are defined at every position inside the superconductor. It is assumed here that the distance over which $B$ and $H$ vary is large compared with the spacing between neighbouring flux lines. The relation between $B$ and $H$ is given by $B=\mu_{0} H$. The current density $\vec{J}$ now is connected to $B$ by

$$
\vec{j}=1 / \mu_{0} \nabla \times \vec{B}
$$

In this formulation the current density $\vec{j}=\nabla x \vec{H}$ is related to all current densities in the bulk of the sample including magnetization currents. ${ }^{4,7}$ In this description, the quantity $H$ will not appear as there is no need for this parameter. As usual, isothermal conditions will be assumed. This means that the temperature dependence of the parameters of the CSM may be neglected as well as thermo-magnetic and galvano-magnetic effects.

The main parameter in the CSM is the critical current density $\vec{j}_{\mathrm{c}},{ }_{1,2}$ which may be a function of the local induction $B$ and of position. This critical current density is connected to the pinning of flux lines and to defects inside the material: it describes the superconducting bulk currents. Reversible surface currents (Meissner currents) are described in terms of a $B_{\text {eq }}\left(B_{\mathrm{a}}\right)$ relation, which gives the flux density just inside a narrow, flux line free, surface layer as a function of the applied external induction $B_{\mathrm{a}}$. When irreversibilities in the surface screening (superheating and supercooling) are also taken into account, 
$B_{\text {eq }}\left(B_{\mathrm{a}}\right)$ is replaced by a $B_{\text {en }}\left(B_{\mathrm{a}}\right)$ relation in increasing field and a $B_{\mathrm{ex}}\left(B_{\mathrm{a}}\right)$ relation in decreasing field respectively. In fact these relations define an induction difference $\Delta B_{\mathrm{s}}$, which is related to the surface current by (1). These surface irreversibilities are often denoted in terms of $\Delta B_{\mathrm{a}}$ (or $\left.\Delta H\right)$ : the maximum variation of the external field which does not give rise to variations of the flux inside the bulk of the superconductor. ${ }^{8}$ In Fig. 1, the flux and current distributions inside a slab are drawn schematically for the case when $j_{\mathrm{c}}$ depends on the $x$-co-ordinate only: $j_{\mathrm{c}}=j_{\mathrm{c}}(x)$. Also the $B_{\mathrm{en}}$ - and $B_{\mathrm{ex}}$-values are indicated. The time dependent behaviour may differ considerably from the quasi-static one because over-critical currents, due to the motion of flux lines, will occur inside the sample. These flux flow effects can be described in the same way as normal currents in terms of a flux flow conductivity $\sigma_{\mathrm{ff}}$. The total current is now given by:

$$
\vec{j}=\vec{j}_{\mathrm{c}}+\sigma_{\mathrm{ff}} \vec{E}
$$

From these three concepts, bulk pinning, surface screening and flux flow, the average induction $B_{\mathrm{av}}$ inside the superconductor can be determined taking into account the dimensions of the sample, its magnetic history and the waveform of the applied magnetic field $B_{\mathrm{a}}(t)$. Since both bulk critical currents and over-critical currents delay flux entry and flux exit, the average induction in increasing field is smaller than $B_{\text {en }}\left(B_{\mathbf{a}}\right)$ :

$$
\begin{aligned}
B_{\mathrm{av}}= & B_{\mathrm{en}}\left(B_{\mathrm{a}}\right)-\Delta B_{\mathrm{p}}\left(B_{\mathrm{a}}, B_{0}-b_{0}\right) \\
& -\Delta B_{\mathrm{ff}}\left(B_{\mathrm{a}}, B_{0}-b_{0}, \mathrm{~d} B_{\mathrm{a}} / \mathrm{d} t\right)
\end{aligned}
$$

in which $\Delta B_{\mathrm{p}}$ indicates the flux screened by bulk currents and $B_{\mathrm{ff}}$ is the delay caused by flux flow $\left(B_{0}-b_{0}\right.$ is the field at which the external field change starts). In decreasing field, (3) transforms to:

$$
B_{\mathrm{av}}=B_{\mathrm{ex}}\left(B_{\mathrm{a}}\right)+\Delta B_{\mathrm{p}}+\Delta B_{\mathrm{ff}}
$$

This average induction $B_{\text {av }}$ as a function of external field $B_{\mathrm{a}}$ is shown schematically in Fig. 2 together with a $B_{\text {en }}\left(B_{\mathrm{a}}\right)$ and a $B_{\text {ex }}\left(B_{\mathrm{a}}\right)$ curve. In increasing field, $B_{\mathrm{aw}}$ is lower than $B_{\text {en }}\left(B_{\mathrm{a}}\right)$ because of flux pinning and flux flow (see (3)). During the time interval with constant external field, the flux flow effects disappear gradually and only $\Delta B_{\mathrm{p}}$ remains. When $B_{\mathrm{a}}$ is decreased again, nothing will happen until $B_{\text {ex }}\left(B_{\mathrm{a}}\right)=B_{\text {en }}\left(B_{0}+b_{0}\right)$. A more comprehensive discussion of this Generalized Critical State Model (GCSM) has been given elsewhere. ${ }^{3,4}$

Inductive techniques. The general principle of inductive techniques is that a sample is placed in an alternating magnetic field. The variations of the average flux density inside the sample are measured by means of a pick-up coil wound around the centre of the sample. Some important characteristics of all inductive techniques are: one, the techniques are non-destructive; two, only variations of the average magnetic induction can be measured; three, the induced voltage represents an average value of the response over the volume of the pick-up coil; and four, the induced voltage due to flux changes in the empty space between pick-up coil and sample has to be compensated.

These points, especially the points two and three have important consequences for the interpretation of the results on type II superconductors. The most important one is that for the interpretation of the results in terms of the

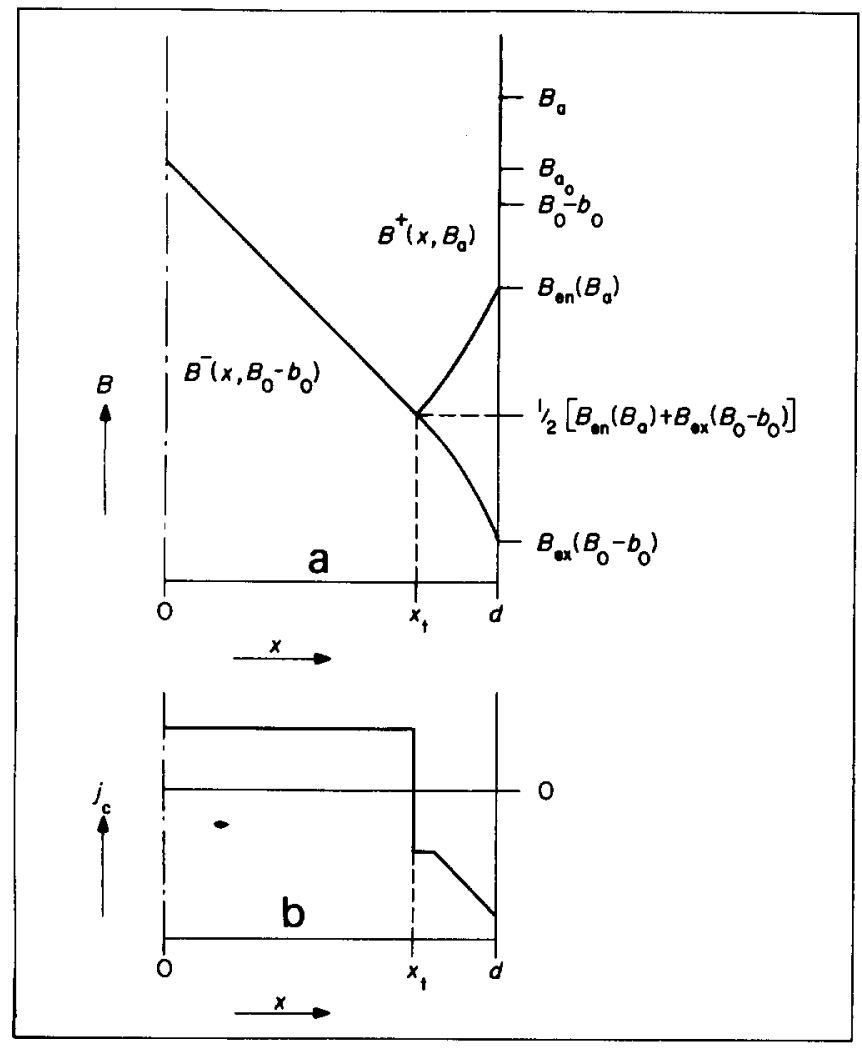

Fig. 1 Flux profiles at two times during the ac cycle; before the increase of the external field starts $\left(B_{\mathrm{a}}=B_{0}-b_{0}\right)$ and for $B_{a} \leqslant B_{0}^{+}$the profile is given by $B^{-}\left(x, B_{0}-b_{0}\right)$. For $B_{\mathrm{a}} \geqslant B_{\mathrm{aO}}$ the profile is given by $B^{-}\left(x, B_{0}-b_{0}\right)$ for $0 \leqslant x \leqslant x_{t}$ and by $B^{+}\left(x, B_{a}\right)$ for $x_{t} \leqslant x \leqslant d$. The corresponding current distribution is also given

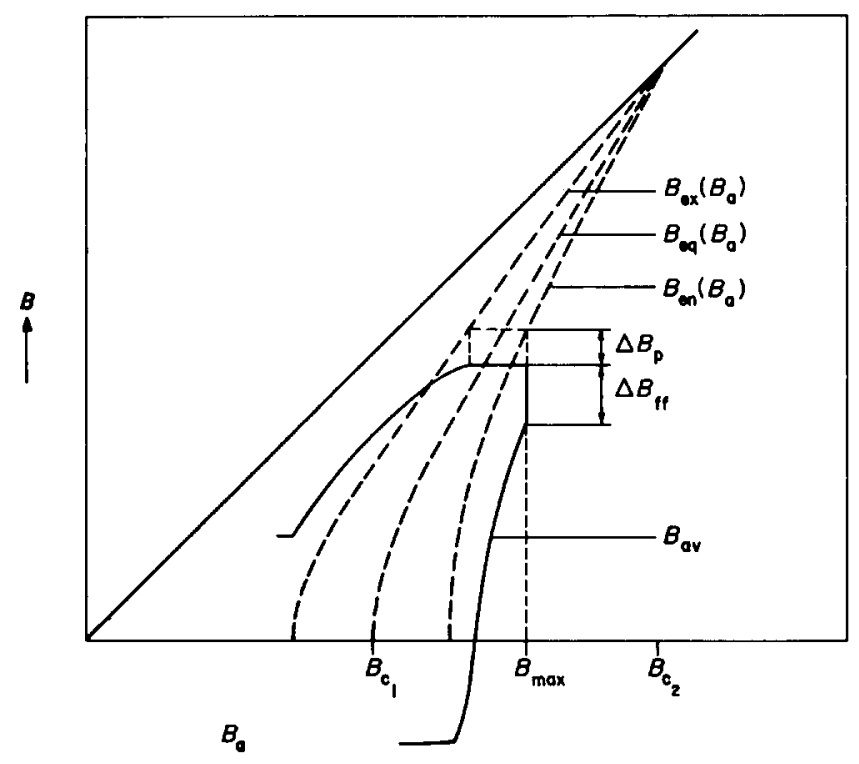

Fig. 2 Schematical $B_{\text {av }}$ vs $B_{\text {a }}$ curve showing the various paramaters of the CSM. The average flux density $\Delta B_{\mathrm{p}}$ screened by the superconducting bulk currents and $\Delta \boldsymbol{B}_{\mathrm{ff}}$ screened by normal currents are also shown

CSM for each of these techniques, assumptions with respect to the parameters of this model have to be made. Assumptions which are usually made are the neglectance of the dependence of $j_{\mathfrak{c}}$ on position and/or magnetic induction, the neglectance of surface currents or the absence of flux flow effects. Of course, the results must be checked for their consistency with the assumptions. The two essential points 
mentioned above, also have consequences for the most suitable experimental situations. Since an average over the volume of the pick-up coil is measured, the geometry of the sample is very important; usually only cylindrical and slabs are used. Due to the elasticity of the flux line lattice ${ }^{9}$ the induced voltage may also depend upon the properties of the superconductor outside the pick-up coil. For this reason the sample must be homogeneous in the direction parallel to the applied field and sufficiently long to avoid end effects. The experiments presented in this paper have been performed in the simple geometry of a slab in a magnetic field parallel to the surface. Also the waveform of the applied magnetic field is of great importance and especially $\mathrm{d} B_{\mathrm{a}} / \mathrm{d} t$, since all induced voltages are directly proportional to this quantity.

In the literature, various methods based on this principle have been proposed to give information on the parameters of the CSM. These techniques can be distinguished into two categories: the $\mathrm{dc}$ and the ac techniques. In the dc case, the external magnetic field is varied quasi-statically; the induced voltage is integrated giving a $B_{\mathrm{av}}$ vs $B_{\mathrm{a}}$ curve or a magnetization curve $M\left(B_{\mathrm{a}}\right)$. With the help of such dc magnetization experiments, Fietz and $W_{e b b^{10}}$ determined the critical current density $j_{\mathrm{c}}$; this determination is, among others, based on the assumption that $j_{\mathrm{c}}$ is independent of position. ac technqiues are a much more powerful instrument for the investigation of type II superconductors. They have already been employed for a long time to investigate the behaviour of normal metals ${ }^{11}$ and paramagnetic salts. ${ }^{12}$ The use of ac techniques for the study of type II superconductors was first proposed by Bean ${ }^{13}$ in 1964 . In these experiments the third harmonic of the induced voltage has been measured and compared to model calculations involving a position independent of critical current density; surface currents are ignored. This type of experiment is usually called harmonic analysis.

Campbel1 ${ }^{14}$ for the first time succeeded in measuring the flux density as a function of position by varying the amplitude of the ac field. In these so-called total flux measurements, however, the critical current density has to be assumed to be independent of $B$ instead of posttion independent. A comparison of the various techniques for the determination of the critical current density has been given by Kroeger et al. ${ }^{15}$

An important distinction between the various ac techniques is that one can either measure a time average of the response signal over one period of the sinusoidal ac field, or observe the waveform of the induced voltage directly. The latter possibility, which often gives more information, was first proposed by Ullmaier ${ }^{8}$ and has also been used by Rollins et al. ${ }^{6}$ in their modification of Campbell's method. A far reaching consequence of this point is that it is no longer necessary to confine oneself to sinusoidal ac fields. This was also first proposed by Ullmaier ${ }^{8}$ who used a triangular waveform giving a constant $\mathrm{d} B_{\mathrm{a}} / \mathrm{d} t$ value. The method presented in this paper is based on the use of trapezoidal ac fields. ${ }^{17,3} \mathrm{~A}$ similar approach was proposed by Eckert and Handstein. ${ }^{18}$

Before discussing the possibilities of trapezoidal ac fields in detail, the response of a very simple superconductor (a slab with a constant critical current density) will be outlined as an illustration. The response of such a superconductor to a trapezoidal waveform (Fig. 3a) is characterized by a linearly increasing voltage until the flux change

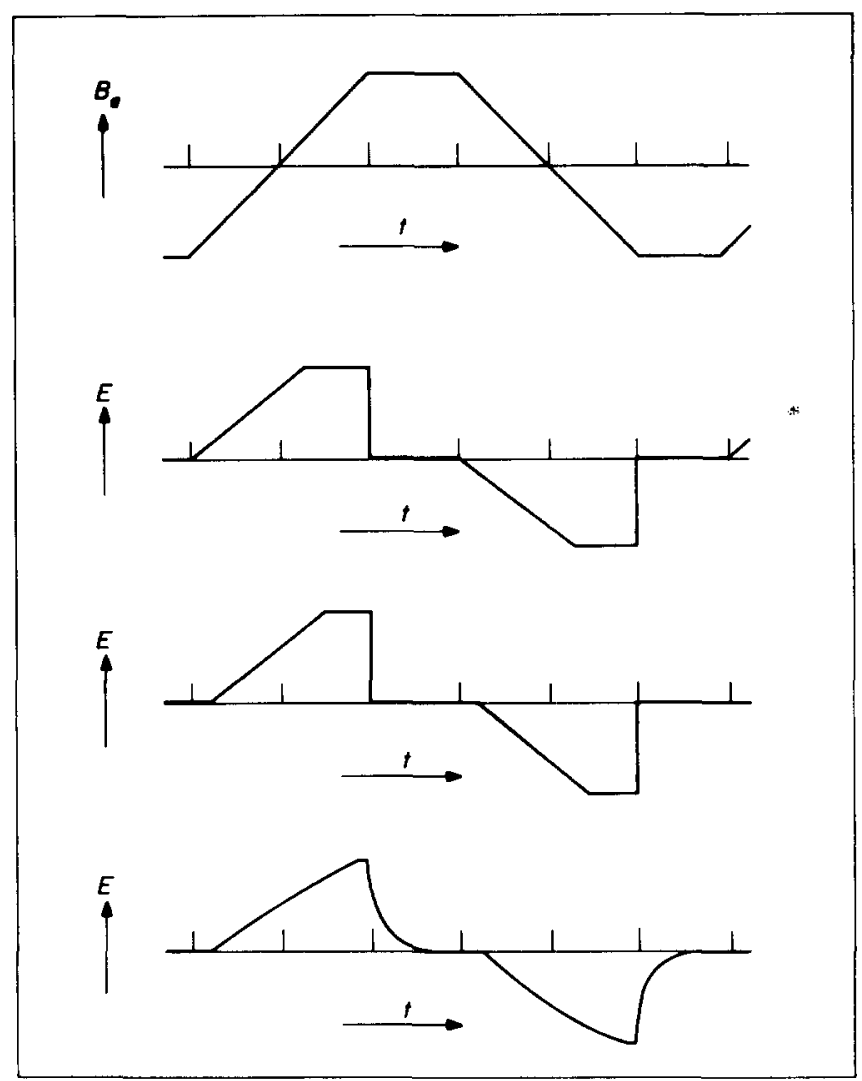

Fig. 3 Schematic response to trapezoidal ac field: a - waveform of the applied field; $b$ - response with $j_{c}=$ constant; $c$ - as b, but with surface screening; $d-$ as $c$, but with flux flow effects

reaches the central plane of the slab (see Fig. 3b). After that the voltage remains constant as long as the external voltage is still varying linearly with time. When the external field is stopped, the voltage drops down to zero again. In Fig. 3c, the influence of an extra surface screening current is shown, giving an interval with zero induced voltage. When flux flow effects are also included, an exponential decay appears when the external field is stopped (see Fig. 3d). Although flux flow effects will also influence the other parts of the induced waveform, they can be studied separately during the constant part of the trapezium. This simple model already indicates the possibilities of trapezoidal ac fields in distinguishing between the various paramaters of the Critical State Model.

$A$ slab in a trapezoidal ac field. As was indicated before, the use of trapezoidal ac magnetic fields provides a method for the separation of the various parameters of the CSM. In this section the determination of the flux distribution inside a superconducting slab will be discussed. The method is related to the technique proposed by Rollins et al. ${ }^{16}$ with the distinction that in their experiments sinusoidal ac fields are used. For a good understanding of the method, it is necessary to study the response of a superconducting slab of width $2 d$ in an ac magnetic field (amplitude $b_{0}$ ) with trapezoidal waveform superposed on a static field $B_{0}$. Both magnetic fields are parallel to the surface of the slab. The analysis is based completely on the validity of the CSM as it was described before. In the derivation below the assumption that flux flow effects may be neglec: ted has been used; this assumption is common to all techniques of the same kind. ${ }^{14,16}$

In the following, only the response in increasing field will be discussed; in decreasing field the derivation is completely 
analogue and only the results will be given. At the minimum of the external field $B_{0}-b_{0}$, the flux density just inside the surface layer is given by $B_{\mathrm{ex}}\left(B_{0}-b_{0}\right)$. When the external field $B_{\mathrm{a}}$ is increased again, no flux will penetrate the sample until the applied field reaches the value $B_{\mathrm{a} 0}^{+}$given by $B_{\text {en }}\left(B_{\mathrm{a} 0}^{+}\right)=B_{\text {ex }}\left(B_{0}-b_{0}\right)$. This interval, during which no penetration takes place, gives information on the irreversibility of the surface currents. After this, flux penetrates to the position $x=x_{t}$. This situation is illustrated in Fig. 1 . In the region $0 \leqslant x \leqslant x_{t}$, the flux distribution will be unclanged:

$$
\begin{aligned}
B^{-}\left(x, B_{\mathrm{a}}\right) & =B_{\mathrm{ex}}\left(B_{0}-b_{0}\right) \\
& +\mu_{0} \int_{x}^{d} j_{\mathrm{c}}\left(B^{-}\left(x^{\prime}, B_{0}-b_{0}\right), x^{\prime}\right) \mathrm{d} x^{\prime} \\
& 0 \leqslant x \leqslant x_{\mathrm{t}}
\end{aligned}
$$

Near the surface, the critical current density has changed sign and the flux profile is given by:

$$
\begin{aligned}
B^{+}\left(x, B_{\mathrm{a}}\right) & =B_{\text {en }}\left(B_{\mathrm{a}}\right) \\
& -\mu_{0} \int_{x}^{d} j_{\mathrm{c}}\left(B^{+}\left(x^{\prime}, B_{\mathrm{a}}\right), x^{\prime}\right) \mathrm{d} x^{\prime} \\
x_{\mathrm{t}} & \leqslant x \leqslant d
\end{aligned}
$$

From these two expressions the average induction inside the sample and the induced voltage can be calculated as a function of applied field. The latter is given by:

$$
\begin{aligned}
E(t) & =\frac{\mathrm{d} B_{\mathrm{a}}}{\mathrm{d} t}\left[\left(d-x_{\mathrm{t}}\right) \frac{\mathrm{d} B_{\mathrm{en}}}{\mathrm{d} B_{\mathrm{a}}}\right. \\
& \left.-\mu_{0} \int_{x_{\mathrm{t}}}^{d} \int_{x}^{d} \frac{\partial j_{\mathrm{c}}\left(B^{+}, x^{\prime}\right)}{\partial B^{+}} \frac{\partial B^{+}}{\partial B_{\mathrm{a}}} \mathrm{d} x^{\prime} \mathrm{d} x\right]
\end{aligned}
$$

Here a second assumption appears: when the critical current density $j_{c}$ may be considered independent of $B$ within the relevant range of $B$-values, the awkward integral term in (7) disappears and a simple expression remains:

$$
E(t)=\left(d-x_{\mathrm{t}}\right) \frac{\mathrm{d} B_{\mathrm{en}}}{\mathrm{d} B_{\mathrm{a}}} \frac{\mathrm{d} B_{\mathrm{a}}}{\mathrm{d} t}
$$

After the flux change has reached the centre plane of the slab $\left(x_{\mathrm{t}}=0\right),(8)$ reduces to:

$$
E_{1}(t)=d \frac{\mathrm{d} B_{\text {en }}}{\mathrm{d} B_{\mathrm{a}}} \frac{\mathrm{d} B_{\mathrm{a}}}{\mathrm{d} t}
$$

So far the fact that a trapezoidal field, ie a linearly increasing field, is applied has not yet been used. The constant value of $\mathrm{d} B_{\mathrm{a}} / \mathrm{d} t$ now means that after the flux front has reached the central plane, the induced waveform is independent of the external field at which the field change started. That this is actually the case can be seen from Fig. 4 . The point at which an individual curve coincides with the envelope curve indicates penetration to the centre of the slab. A consequence of this fact is that (9) directly gives an expression for $\mathrm{d} B_{\text {en }} / \mathrm{d} B_{\mathrm{a}}$. By dividing (8) through (9) one gets:

$$
\frac{E}{E_{1}}=\frac{d-x_{\mathrm{t}}}{d}
$$

giving a direct measure for the position of the flux front. It should be borne in mind that it is a direct consequence of the linearity of the external field change that the variation of surface currents, denoted by $\mathrm{d} B_{\text {en }} / \mathrm{d} B_{\mathrm{a}}$ may be easily taken into account. If sinusoidal ac fields are used this is not possible because $\mathrm{d} B_{\mathrm{a}} / \mathrm{d} t$ varies continuously and even becomes zero. The local induction at the position $x_{t}$ is now given by

$$
\begin{aligned}
B\left(x_{\mathrm{t}}, B_{\mathrm{a}}\right) & =1 / 2\left\{B_{\mathrm{en}}\left(B_{\mathrm{a}}\right)+B_{\mathrm{ex}}\left(B_{0}-b_{0}\right)\right\} \\
& +1 / 2 \mu_{0} \int_{x_{\mathrm{t}}}^{d}\left[j_{\mathrm{c}}\left\{B^{-}\left(x^{\prime}-b_{0}\right), x^{\prime}\right\}\right. \\
& \left.-j_{\mathrm{c}}\left\{B^{+}\left(x^{\prime}, B_{\mathrm{a}}\right), x^{\prime}\right\}\right] \mathrm{d} x^{\prime}
\end{aligned}
$$

If $j_{\mathrm{c}}$ may be assumed independent of $B$ within the relevant field range, the integral term in (11) vanishes and the equation can be written:

$$
\begin{aligned}
B\left(x_{\mathrm{t}}, B_{\mathrm{a}}\right) & =B_{\mathrm{ex}}\left(B_{0}-b_{0}\right) \\
& +1 / 2 \int_{B_{\mathrm{a} 0}^{+}}^{B_{\mathrm{a}}} \frac{\mathrm{d} B_{\mathrm{en}}}{\mathrm{d} B_{\mathrm{a}}} \mathrm{d} B_{\mathrm{a}}
\end{aligned}
$$

This quantity can be determined from the envelope curve
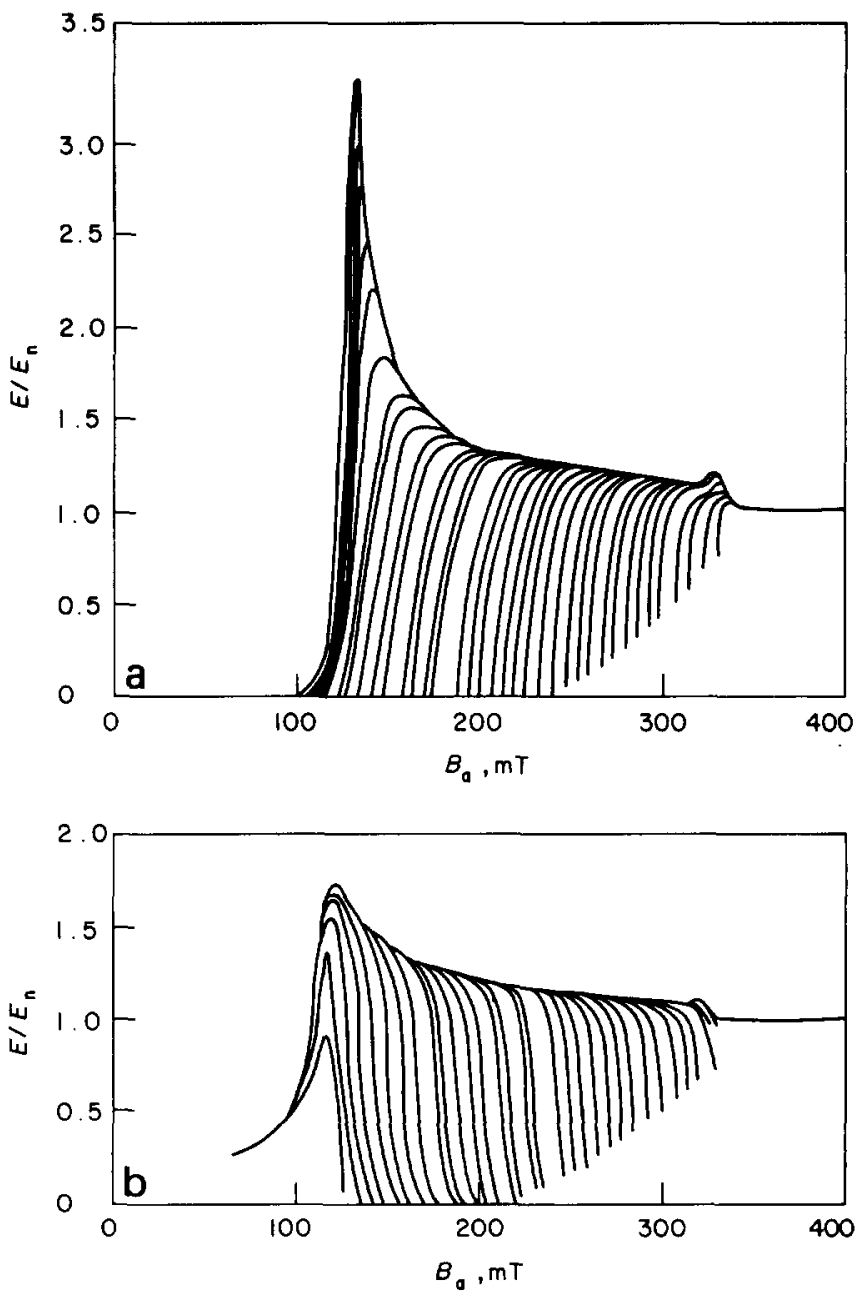

Fig. 4 Experimental $E$ vs $B_{\mathrm{a}}$ curves for various values of the static field $B_{0}$ for increasing (a) and decreasing field (b) for the Nb-slab. The envelope curves can be seen clearly $\left(b_{0}=32 \mathrm{mT}, \mathrm{d} B_{\mathrm{a}} / \mathrm{d} t=\right.$ $0.67 \mathrm{~T} \mathrm{~s}^{-1}$ ) 
$E_{1}\left(B_{\mathrm{a}}\right)$. In most cases, however, a first order Taylor expansion of $B_{\text {en }}\left(B_{\mathrm{a}}\right)$ is sufficient:

$$
\begin{aligned}
B\left(x_{\mathrm{t}}, B_{\mathrm{a}}\right) & =B_{\text {ex }}\left(B_{0}-b_{0}\right) \\
& +1 /\left.2\left(B_{\mathrm{a}}-B_{\mathrm{a} 0}^{+}\right) \frac{\mathrm{d} B_{\mathrm{en}}}{\mathrm{d} B_{\mathrm{a}}}\right|_{B_{\mathrm{a}}=B_{\mathrm{a} 0}^{+}}
\end{aligned}
$$

which is proportionai to $B_{\mathrm{a}}$.

It can be concluded from (8) and (13) that if $\mathrm{d} B_{\text {en }} / \mathrm{d} B_{\mathrm{a}}$ is independent of $B_{\mathrm{a}}$ within the field range during which the penetration of the flux front from surface to central plane takes place, the shape of the flux profile at which the field variation started $\left(B_{\mathrm{a}}=B_{0}-b_{0}\right)$ can be seen directly from the $E$ vs $B_{\mathrm{a}}$ curve by turning this curve through 90 degrees. When $\mathrm{d} B_{\text {en }} / \mathrm{d} B_{\mathrm{a}}$ is not a constant, the envelope curve $E_{1}\left(B_{\mathrm{a}}\right)$ (see (9) and Fig. $\left.4 \mathrm{a}\right)$ has to be determined and calculations according to (10) and (13) are necessary. In this case the line $E / E_{1}=0$ indicates the surface of the superconductor while the envelope curve $\left(E / E_{1}=1\right)$ may be considered as the central plane of the slab. Examples of flux profiles obtained in this way are given later. During the decreasing interval of the ac field, the flux distribution at the maximum of the ac field $\left(B_{\mathrm{a}}=B_{0}+b_{0}\right)$ can be determined in a similar way. Equation (13) now becomes:

$$
\begin{aligned}
B\left(x_{\mathrm{t}}, B_{\mathrm{a}}\right) & =B_{\mathrm{en}}\left(B_{0}+b_{0}\right) \\
& +1 /\left.2\left(B_{\mathrm{a}}-B_{\mathrm{a} 0}^{-}\right) \frac{\mathrm{d} B_{\mathrm{ex}}}{\mathrm{d} B_{\mathrm{a}}}\right|_{B_{\mathrm{a}}=B_{\mathrm{a} 0}^{-}}
\end{aligned}
$$

in which $B_{\mathrm{a} 0}^{-}$is given by $B_{\mathrm{ex}}\left(B_{\mathrm{a} 0}^{-}\right)=B_{\mathrm{en}}\left(B_{0}+b_{0}\right)$. If a cylinder with radius $R$ is used instead of a slab, ${ }^{16}(8)$ is transformed into:

$$
E(t)=\frac{1}{2 R}\left(R^{2}-r_{\mathrm{t}}^{2}\right) \frac{\mathrm{d} B_{\mathrm{en}}}{\mathrm{d} B_{\mathrm{a}}} \frac{\mathrm{d} B_{\mathrm{a}}}{\mathrm{d} t}
$$

in which $r_{\mathrm{t}}$ is the position to which the flux change has penetrated.

The foregoing derivation clearly indicates that the most convenient experimental conditions for the investigation of flux distributions in type II superconductors are those in which the response of a superconducting slab to a linearly varying magnetic field is measured. In that case, only two assumptions with respect to the parameters of the CSM have to be made. These assumptions are: one, flux flow effects may be neglected; and two, the critical current density is independent of $B$ at least in the field range in which the flux front has not reached the centre plane. ${ }^{9}$

Whether these assumptions are satisfied, and therefore whether the interpretation of the experimental results is correct, must be checked for every individual sample separately.

Separation of loss contributions. ac losses in type II superconductors are caused by three different mechanisms: bulk pinning, surface screening and flux flow. In the previous section it was demonstrated how the flux distribution, and from this the critical current density, can be determined by means of a direct observation of the waveform of the induced voltage. This, however, gives no in- formation with respect to surface screening currents and flux flow effects. The latter phenomenon can be studied separately during those intervals of the ac field during which this field is constant. In these intervals the contribution of the two other (quasi-static) mechanisms disappears and only flux flow remains. The value of the flux flow conductivity $\sigma_{\text {ff }}$ can be determined from the time constant of the decay curve or from the amount of flux which still enters or leaves the sample when the external field is kept constant. A detailed discussion of this technique in the case of normal conducting metals has been given elsewhere. ${ }^{20,21}$

So far both $j_{\mathrm{c}}$ and $\sigma_{\mathrm{ff}}$ are determined as a function of the externally applied induction $B_{\mathrm{a}}$ instead of the internal induction. Also with respect to the surface screening current, the only quantity known so far is the amount of flux which can be screened at a certain external field. Since an inductive technique is used, only variations of the average flux density $B_{\mathrm{av}}$ inside the sample can be measured. Therefore it is impossible to determine the value of $B_{\mathrm{av}}$ itself from one single measurement. The most simple method for the determination of $B_{\mathrm{av}}$ as a function of $B_{\mathrm{a}}$ is to make a hysteresis loop of large amplitude. In this case there are two possible references which can be used to obtain the correct value of $B_{\mathrm{av}}$ : one, in the normal case $\left(B_{\mathrm{a}}>B_{\mathrm{c} 2}\right)$, hysteresis is caused by the normal resistivity which is determined by $\mathrm{d} B_{\mathrm{a}} / \mathrm{d} t$ only. In this case the mean value of $B_{\mathrm{av}}$ in increasing and decreasing field equals $B_{\mathrm{a}}$. Two, the large hysteresis loop is symmetrical with respect to the line $B_{\mathrm{av}}=-B_{\mathrm{a}}$, ie the value of $B_{\mathrm{av}}$ for $B_{\mathrm{a}}=0$ in increasing and decreasing fields, respectively, have the same absolute value but a different sign. Form this the $B_{\mathrm{av}}=0$ level can be determined.

Instead of one large amplitude hysteresis loop, $B_{\text {av }}$ can also be determined from a large number of small amplitude loops measured at different static fields. In this case, it is necessary that in each individual loop the flux change reaches the centre plane of the slab (the envelope curve) and that these parts of the curves overlap. This is, however, also a necessary condition for the construction of the $E_{1}\left(B_{\mathrm{a}}\right)$ curves. This latter possibility is preferred because it also ensures that all experiments are done at the same $\mathrm{d} B_{\mathrm{a}} / \mathrm{d} t$. The $B_{\mathrm{av}}$ vs $B_{\mathrm{a}}$ curve obtained in this way can be used for a plot of $j_{\mathrm{c}}$ or $o_{f f}$ versus average internal induction.

These $B_{\mathrm{av}}$ vs $B_{\mathrm{a}}$ curves are also the starting point for the determination of $B_{\text {en }}\left(B_{\mathrm{a}}\right)$ and $B_{\text {ex }}\left(B_{\mathrm{a}}\right)$. As can be seen from (3) the difference between $B_{\mathrm{av}}\left(B_{\mathrm{a}}\right)$ and $B_{\mathrm{ex}}\left(B_{\mathrm{a}}\right)$ consists of two contributions $\Delta B_{\mathrm{p}}$ and $\Delta B_{\mathrm{ff}}$. This is illustrated in Fig. 5 where a small hysteresis loop is plotted together with some

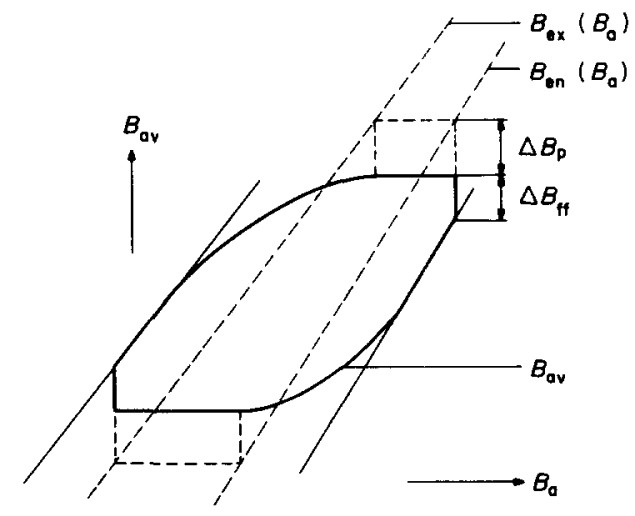

Fig. 5 Schematical representation of a small hysteresis loop together with parts of the overall hysteresis curve 
parts of the envelope $B_{\mathrm{av}}$ vs $B_{\mathrm{a}}$ curves in increasing and decreasing field respectively. The flux flow contribution $\Delta B_{\mathrm{ff}}$ be determined for a large number of $B_{\mathrm{a}}$ values by integrating the decay curve with respect to time. The flux pinning contribution $\Delta B_{\mathrm{p}}$ can be determined from the flux distribution; in the case of a constant critical current density throughout the bulk of the slab it follows: $\Delta B_{\mathrm{p}}=$ $1 / 2 \mu_{0} j_{\mathrm{c}} d$. So in this way the $B_{\text {en }}\left(B_{\mathrm{a}}\right)$ relation can be determined according to (3). In a similar way, $B_{\mathrm{ex}}\left(B_{\mathrm{a}}\right)$ can be determined according to (4). The results should be in accordance with the length of the $E=0$ parts in the $E$ vs $B_{\mathrm{a}}$ curves.

In this way the various parameters of the CSM can be determined separately by performing a large number of experiments with trapezoidal ac fields of small amplitude superposed on a static field. Some results of these experiments will be shown later.

Verification of the experimental technique. The experimental technique presented in the two preceding sections can be verified with the help of numerical model calculations. These calculations have been performed for quasi-static conditions with a numerical technique similar to the one used by Walmsley. ${ }^{22}$ A detailed description of this technique and results on induced voltage waveforms, hysteresis loops and ac losses will be presented elsewhere. ${ }^{23}$ Here we will restrict ourselves to a verification of the experimental procedure, ie to the question of whether calculated induced voltage waveforms, when treated in the same way as the experimental curves, reproduce the assumed $j_{\mathrm{c}}(B, x), B_{\mathrm{en}}\left(B_{\mathrm{a}}\right)$ and $B_{\text {ex }}\left(B_{\mathrm{a}}\right)$ relations.

For this purpose two types of calculations have been performed. In the first place a position dependent, critical current density has been assumed according to:

$$
j_{\mathrm{c}}(B, x)=j_{0}(x) f(B)=j_{0}(x) /\left(1+|B| B_{1}\right)
$$

with

$$
\begin{aligned}
& j_{0}(x)=j_{0}+\left\{x-\left(d-d_{\mathrm{s}}\right)\right\} j_{1} \\
& d-d_{\mathrm{s}} \leqslant x \leqslant d \\
& j_{0}(x)=j_{0} \\
& 0 \leqslant x \leqslant d-d_{\mathrm{s}}
\end{aligned}
$$

Equations (16) and (17) denote a linearly increasing $j_{\mathbf{c}}$ $\left(\partial j_{c} / \partial x=j_{1}\right)$ in a layer of thickness $d_{s}$; for the $B$-dependence a Kim.like relation has been assumed. ${ }^{24}$ Surface currents have been neglected. A comparison between the flux distribution determined according to the experimental procedure and a flux distribution calculated directly is shown in Fig. 6. Although the shape of the 'measured' flux distribution is in good agreement with the original one, a relative systematic error $\epsilon$ of approximately 5\% arises. This error increases for smaller values of the external field. It may be explained from the neglect of the integral terms in (7) and (11) which are related to $\partial j_{c} / \partial B$. An estimate of the relative error on the basis of a large number of calculations yields:

$$
\epsilon<\left.\mu_{0} d \frac{\partial j_{\mathrm{c}}}{\partial B}\right|_{B_{0}-b_{0}}
$$

Only in the field region around $B=0$ have larger deviations

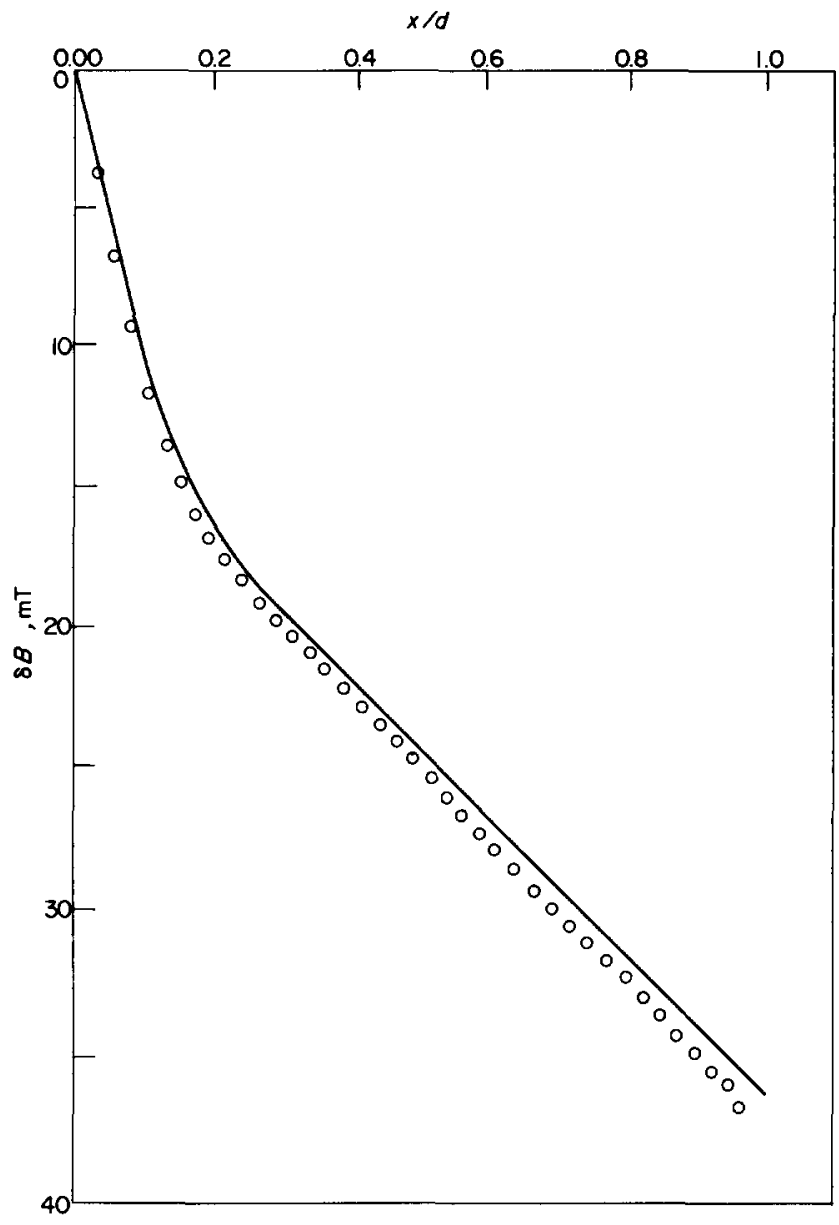

Fig. 6 Comparison of a calculated flux distribution (drawn line) and a distribution determined from the appropriate voltage waveform in increasing field $\left(B_{0}=500 \mathrm{mT} ; b_{0}=90 \mathrm{mT}\right)$. The position dependent $j_{\mathrm{c}}$ is given by $(16)$ and $(17) . v_{0}=0.637 \times 10^{6} \mathrm{~A} \mathrm{~cm}-2$; $B_{1}=10 \mathrm{mT} ;\left(d-d_{s}\right) / d=0.20 ; j_{1}=4 \mu_{0} / d_{s}$

been found due to the change in sign of the integral terms in (7) and (11) in this region.

Secondly, calculations have been performed with a position independent $j_{\mathrm{c}}(B)$ but including a $B_{\text {en }}\left(B_{\mathrm{a}}\right)$ and a $B_{\text {ex }}\left(B_{\mathrm{a}}\right)$ relation. For the latter relations, two curves have been used which were determined experimentally from a $\mathrm{Nb}-$ $50 \% \mathrm{Ti}$ slab. ${ }^{25}$ The quantity $\Delta B_{\mathrm{p}}$ has been determined from the calculated voltage waveforms and combined with the envelope hysteresis loop. The resulting $B_{\text {en }}\left(B_{\mathrm{a}}\right)$ and $B_{\text {ex }}$ $\left(B_{\mathrm{a}}\right)$ relations are in good agreement with the assumed curves as can be seen from Fig. 7 .

It may be concluded that the experimental technique presented in this paper indeed allows the separate determination of the critical current density as well as the surface screening currents. The determination of the conductivity $\sigma$ from the decay curves has already been verified elsewhere. ${ }^{21}$

Trapezoidal ac fields. Preliminary to the discussion of the technical aspects of the method and the experimental results, it is useful to summarize the various advantages of the appliance of trapezoidal ac fields. This partly affects some issues which have already been treated, but a few other positive points will also be mentioned. There are eight advantages for the use of trapezoidal ac fields:

It provides a relatively simple method for determining the flux distribution inside the superconductor. This technique is closely related to the methods of Campbel1 ${ }^{14}$ and espec- 


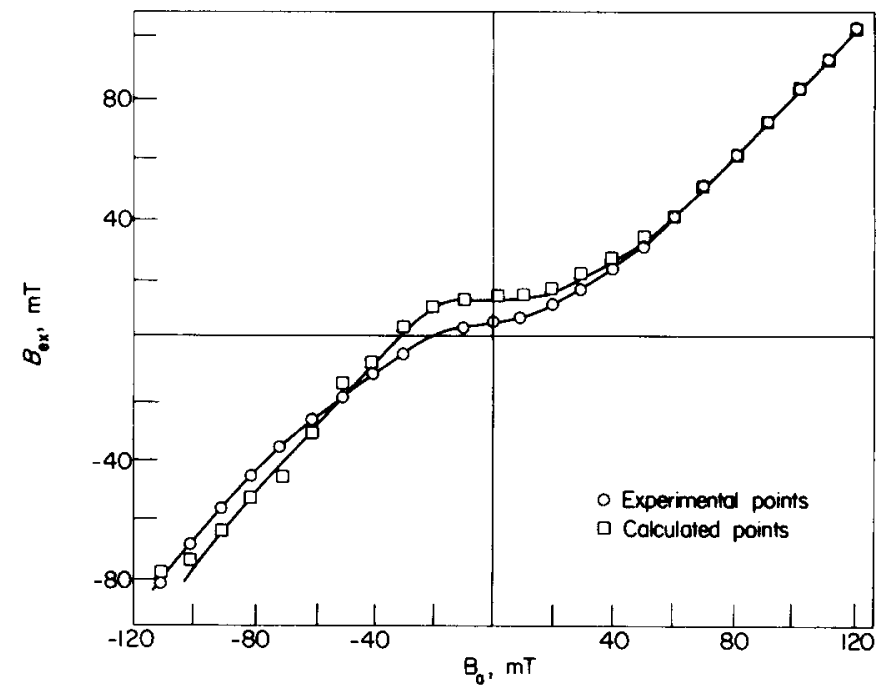

Fig. $7 B_{\text {en }}\left(B_{\mathrm{a}}\right)$ and $B_{\text {ex }}\left(B_{\mathrm{a}}\right)$ curves for $\mathrm{Nb}-50 \% \mathrm{Ti}$. The points have been obtained according to $(3)$ and $(4)\left(b_{0}=180 \mathrm{mT}\right.$; $-300<B_{0}<+300 \mathrm{mT} ; \mathrm{d} B_{\mathrm{a}} / \mathrm{d} t=14.9 \mathrm{~T} \mathrm{~s}^{-1}$. Introduction of the experimental curves in the numerical calculation and applying (3) and (4) to the calculated voltage waveforms leads to the $B_{\text {en }}$ and $B_{\text {ex }}$ values denoted by $\square$

ially Rolling, Küpfer and Gey. ${ }^{16}$ This point has been discussed extensively previously.

Trapezoidal ac fields provide an accurate method for the determination of the conductivity in normal metals as well as type II superconductors. ${ }^{21}$

Direct observation of the induced waveform leads to a separation of the contributions of the various parameters of the CSM to the ac losses. In this the critical current density $j_{\mathrm{c}}$, the surface screening relations $B_{\mathrm{en}}\left(B_{\mathrm{a}}\right)$ and $B_{\mathrm{ex}}\left(B_{\mathrm{a}}\right)$ and the flux flow resistivity $\sigma_{\mathrm{ff}}$ can be determined separately.

The rate of change of the external field $\mathrm{d} B_{\mathrm{a}} / \mathrm{d} t$, which is one of the decisive variables of the induced waveform, only has some distinct values instead of a continuous variation in the case of a sinusoidal field.

A consequence of this is that even at the peak of the ac field, $\mathrm{d} B_{\mathrm{a}} / \mathrm{d} t$ has a large value. When sinusoidal fields are used, $\mathrm{d} B_{\mathrm{a}} / \mathrm{d} t=0$ at the peak of the ac field, thereby masking the behaviour of the superconductor in that region.

The response signal of an empty coil to a trapezoidal ac field contains discontinuities at the corners of the trapezium. In the case of superconductors, such discontinuities have never been observed at those corners of the trapezium where the external field change starts. Therefore, the use of trapezoidal ac fields provides a very clear compensation criterion: the absence of discontinuities at those corners.

While a sinusoidal ac field is determined by two parameters (frequency and amplitude), three independent variables can be distinguished in the trapezoidal waveform: amplitude, $\mathrm{d} B_{\mathrm{a}} / \mathrm{d} t$ and repetition rate. This latter quantity may be important when heating of the sample plays a role. It also provides the possibility to let the flux flow effects disappear completely before the next field change starts.

Additional information with respect to the flux flow properties of the superconductor can be obtained when a socalled double trapezoidal waveform is applied (see Fig. 8). Thus flux flow effects can be studied in two different situations: firstly the flux penetration after the external field is kept constant, and secondly the delayed flux en- trance when the external field is changed again in the same direction.

According to the generalized CSM, the response in both cases should be identical, but some preliminary results indicate minor deviations. In sinusoidal or single trapezoidal fields these effects cannot be seen since the flux flow behaviour at the beginning of the external field sweep is always masked by the reversal of critical currents. A typical response curve is shown in Fig. 8. In this work, however, no further discussion of these results will be given.

\section{The measuring equipment}

General description. In this section, the experimental equipment designed for the measurement of the behaviour of superconductors in ac magnetic fields of trapezoidal waveform is described. A diagram of the complete equipment is given in Fig. 9. A very prominent role in this scheme is played by the trapezium generator which not only provides the appropriate voltage waveform for the ac field but also controls the whole registration of the signal. The signal is digitalized and transmitted to the computer. Because of the synchronization between generated ac waveform and sampling process, the analysis of the signal is very simple and accurate. With the same apparatus, the double trapezoidal waveform can also be generated. Although the equipment is designed for trapezoidal-like ac

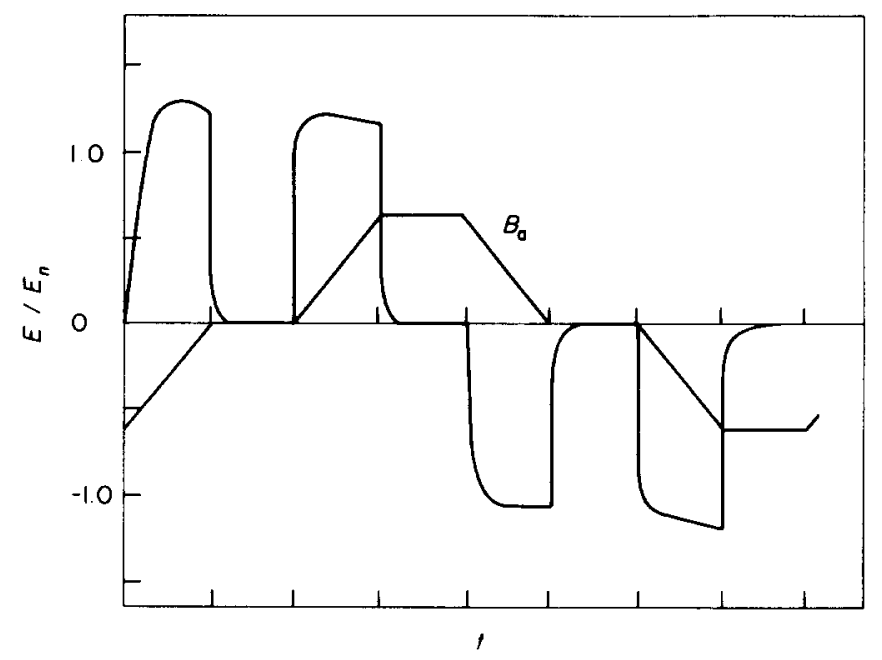

Fig. 8 A typical response curve of a $\mathrm{Nb}$-slab in a double trapezoidal ac field. Also the waveform of the applied field $B_{a}$ is shown $\left(B_{0}=216 \mathrm{mT}, b_{0}=64 \mathrm{mT}, \mathrm{d} B_{\mathrm{a}} / \mathrm{d} t=8 \mathrm{~T} \mathrm{~s}^{-1}\right.$

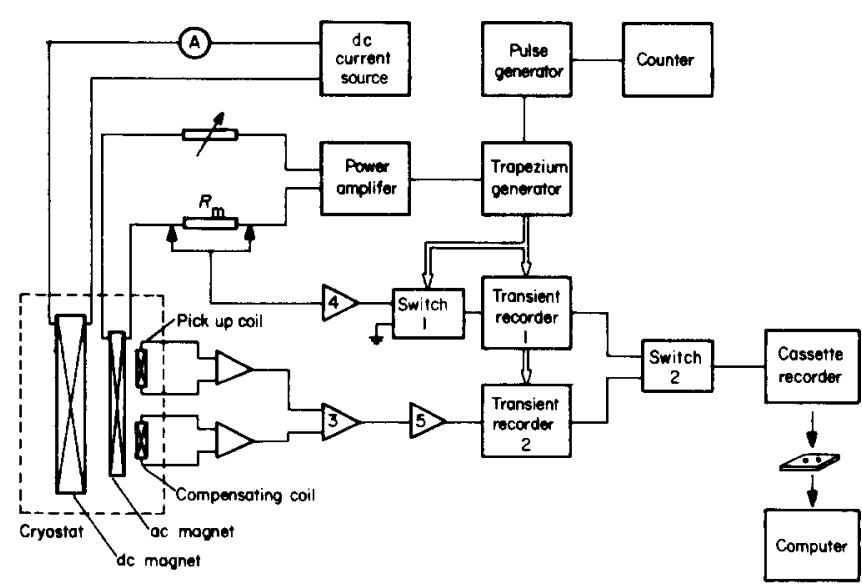

Fig. 9 Block diagram of the experimental equipment. The triangles indicate signal amplifiers; the numbers are referred to in the text 


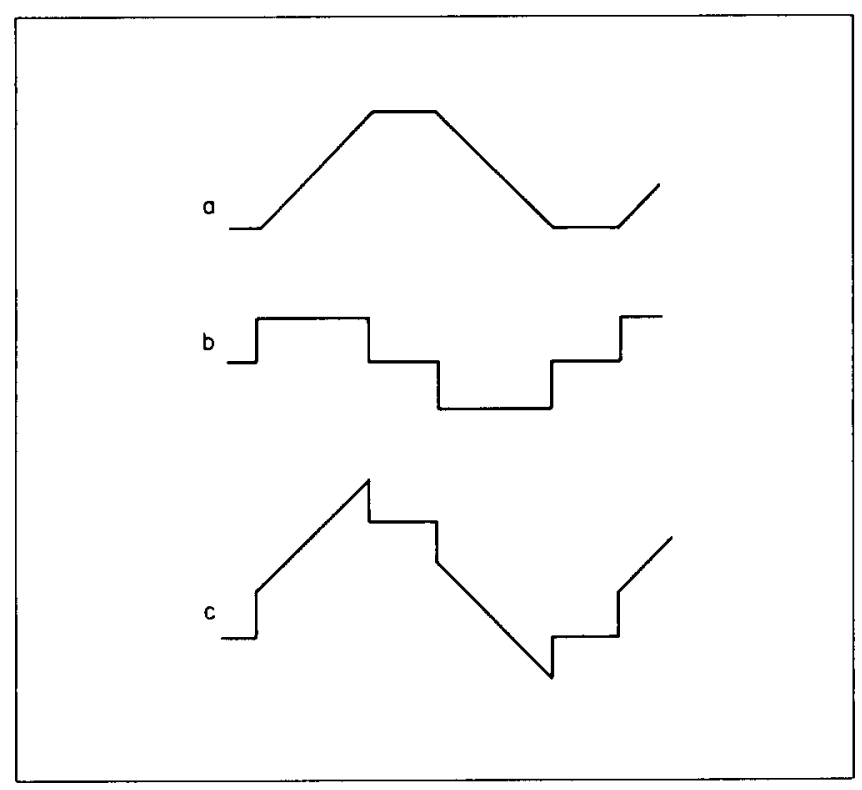

Fig. 10 Required voltages waveforms for a trapezoidal current. $a$ - voltage for a resistance; $b$ - voltage for a self-inductance; c - voltage for a series circuit of resistance and coil

fields, other waveforms (for instance sinusoidal) can be supplied simply by replacing the generator. A consequence, of course, is the loss of the synchronization.

The magnetic field is produced by a trapezoidally varying current through the ac field coil. This current is supplied by an Amcron M600 power amplifier, capable of providing ac currents of up to $35 \mathrm{~A}(20 \mathrm{~A} \mathrm{dc})$, together with ac voltages of between $\pm 100 \mathrm{~V}( \pm 60 \mathrm{~V} \mathrm{dc})$ in the frequency range up to $100 \mathrm{kHz}$. Two resistors are placed in series with this coil: a $0.1 \Omega$ resistance $R_{\mathrm{m}}$ to measure the coil current, and a variable resistance to match the output impedance of the power amplifier. The amplifier is protected against high induction voltages by a crow-bar (not shown in Fig. 9) which short-circuits the amplifier when the voltage exceeds $100 \mathrm{~V}$. The voltage waveform necessary to produce a perfect trapezoidally varying current through a circuit with a resistance in series with a coil consists of two components: a trapezoidal voltage together with its derivative added in the correct ratio for the resistive and inductive component of the circuit respectively (see Fig. 10). This waveform is provided by the trapezium generator, which will be discussed in detail later.

In some experiments the ac coil is placed in a $2 \mathrm{~T}$ dc magnet; the dc current is provided by a HP $6260 \mathrm{~B}$ current supply. The response signal is measured with a pick-up coil and after compensation for the unfilled part of this coil, is sampled by means of a transient recorder. The current waveform is also recorded in this way. Both digitalized signals are then transmitted to the computer with the help of a cassette recorder and analysed. An analysis of the frequency characteristics of the equipment results in a bandwidth of $100 \mathrm{kHz}$. In the rest of this section the coil system, the compensation criterion and the signal processing will be discussed.

The coil system. For the understanding of the ac behaviour of type II superconductors, experiments in large amplitude ac fields as well as in small amplitude ac fields superimposed on a dc field are of interest. The small amplitude ac fields are generated in a small superconducting coil; data on this coil are given in Table 1 together with the data on the other ac coils. The influence of the presence of the $\mathrm{dc}$ coil on the ac field was less than $1 \%$. Large amplitude experiments have been performed in a coil with the same diameter so that the samples can be interchanged easily; the number of turns, however, is much larger. The frequency and amplitude range in which experiments can be done is limited by the properties of the superconducting wire and the cooling conditions. For frequencies below about $2 \mathrm{~Hz}$, the performance is governed by the critical current density of the superconducting wire. With the coil used in our experiments (coil 2), amplitudes of up to $0.5 \mathrm{~T}$ can be obtained at low frequencies, ie for niobium samples the external field can be swept from below $-B_{\mathrm{c} 2}$ to above $+B_{\mathrm{c} 2}$ within $0.1 \mathrm{~s}\left(\mathrm{~d} B_{\mathrm{a}} / \mathrm{d} t=\right.$ $10 \mathrm{~T} \mathrm{~s}^{-1}$ ). At higher frequencies the dissipation inside the coil and the cooling conditions are decisive for the destruction of superconductivity and the maximum amplitude is much less $(340 \mathrm{mT}$ at $10 \mathrm{~Hz}$ and $120 \mathrm{mT}$ at $100 \mathrm{~Hz})$. A similar behaviour was also observed in earlier experiments on other superconducting coils. ${ }^{26}$

In most cases the behaviour of the superconducting sample does not influence the current through the ac coil significantly even at phase transitions. However when very fast phase transitions, ie flux jumps, occur inside the sample, the current in the coil is seriously affected. The $L C$ circuit consisting of the self inductance $L$ and the parallel capacity $C$ of the coil is energized and large oscillations are observed. This effect is greatly reduced by replacing the ac coil during these experiments by another coil with superconducting filaments in a copper matrix. The eddy currents induced in this matrix considerably decrease the quantity of the $L C$ circuit and as a consequence reduce the oscillations. The measuring coil consists of 200 turns of copper wire with a diameter of $0.025 \mathrm{~mm}$ wound close around the middle section of the sample. The compensation coil is wound from 130 turns of the same wire. Both coils are wound in one layer in order to reduce the parallel capacity of the coils as much as possible. For all our samples the cut-off frequency of the pick-up coils is above $2 \mathrm{MHz}$ which means that the observed waveforms are not influenced by the coil system.

Compensation. An essential aspect, common to all inductive techniques, is the necessity of compensation of the induced voltage for the contribution of the empty space surrounding

Table 1. Parameters of the coil system

\begin{tabular}{lcccccl}
\hline Type & $\begin{array}{l}\text { Length, } \\
\mathrm{mm}\end{array}$ & $\begin{array}{l}\text { Diameter, } \\
\mathrm{mm}\end{array}$ & $\begin{array}{l}\text { No of } \\
\text { turns }\end{array}$ & $\begin{array}{l}\text { No of } \\
\text { layers }\end{array}$ & $\begin{array}{l}\text { Diameter } \\
\text { of wire, } \\
\mathrm{mm}\end{array}$ & Type of wire \\
\hline 1. small ac coil & 50 & 7 & 1280 & 2 & 0.05 & Niomax CN A61/05 \\
2. large ac coil & 80 & 7 & 16000 & 16 & 0.05 & Niomax CN A61/05 \\
3. large ac coil & 100 & 30 & 2820 & 7 & 0.20 & Vacuumschmelze F60, 0.20 \\
\hline
\end{tabular}


the sample. The compensating signal is obtained from a second pick-up coil, placed inside the ac field coil but at some distance from the sample. The signals induced in both pick-up coils are amplified by means of two chopper amplifiers (see Fig. 9, numbers 1 and 2) and then subtracted with the help of a third amplifier (number 3 ). The amplification of amplifier 2 is adjusted in such a way that the output signal balances the response of the unfilled part of the measuring coil. This compensation is usually performed in a small ac field at zero static field; in the s situation no flux penetrates the sample and the induced signal is zero. Compensation is of extreme importance when the behaviour of type II superconductors in the vicinity of the field of first penetration is studied. In these experiments, the response of the sample is very small and even a minor variation of the compensation will change the observed waveform drastically.

In the literature a number of authors ${ }^{2729}$ have presented results in which ac losses are observed at fields well below the field of first penetration. This effect is ascribed to flux penetration in irregularities at the surface..$^{30,31}$ The study of this phenomenon is of great significance for the application of superconductors in, for instance, ac transport cables. ${ }^{32}$ In this case, the criterion for correct compensation mentioned above cannot be used anymore and a better criterion has to be found. In most experiments with sinusoidal fields, the criterion $\mathrm{d} \phi / \mathrm{d} t=0$ at the top of the ac field is used for compensation. This implies an extra assumption with respect to the behaviour of the superconductor. The application of trapezoidal ac fields offers an additional criterion: the absence of discontinuities in the compensated signal. Here also an extra assumption with respect to the behaviour of the sample is involved, but it is much less stringent. In fact, experiments with trapezoidal ac fields on various samples $\left(\mathrm{Nb}, \mathrm{Nb}_{3} \mathrm{Sn}\right)$ show that the assumption $\mathrm{d} \phi / \mathrm{d} t=0$ at the peak of the ac field is not justified. This can be seen in Fig. 11 in which even in the time intervals of constant $B_{\mathrm{a}}$, flux changes are observed. The occurrence of these induced voltages cannot be explained by Melvilles theory. ${ }^{31}$

A second important feature of the use of this compensation criterion is that it can be used in the whole field range from zero to $B_{\mathrm{c} 2}$, whereas the other criteria are limited to fields well below $B_{\mathrm{c} 1}$. Especially in the vicinity of $B_{\mathrm{c} 1}$, where large surface screening currents are induced inside the sample, these criteria do not guarantee correct compensation due

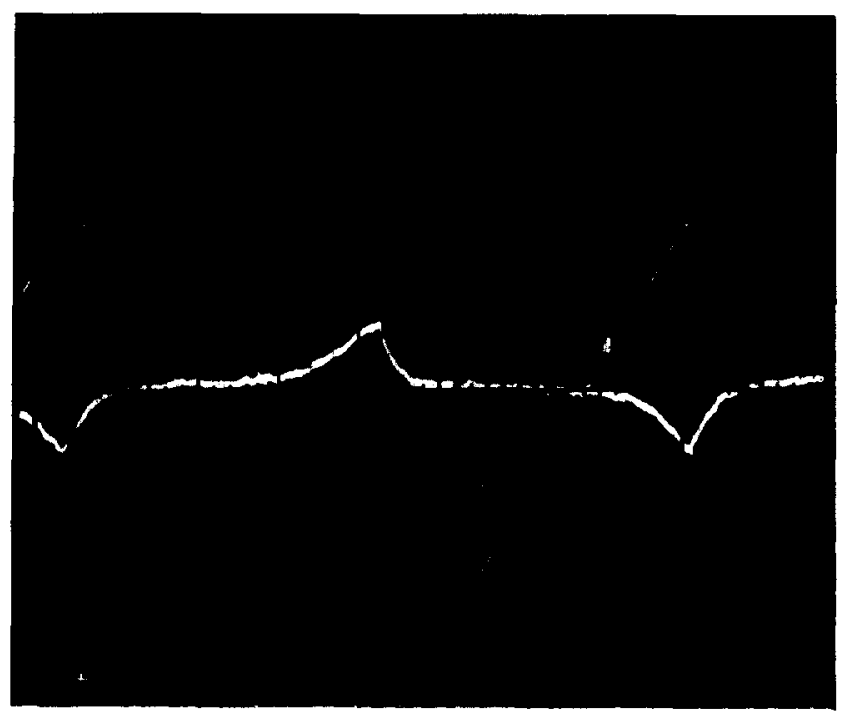

Fig. 11 Induced voltage waveform in the Meissner state of $\mathrm{Nb}$ $\left(b_{0}=100 \mathrm{mT}\right)$. The field of first penetration of $\mathrm{Nb}$ is $110 \mathrm{mT}$ to the demagnetization of the sample. In this case, the field change just outside the sample which is picked up by the measuring coil differs from the flux variation further away which is used for compensation. This is confirmed by the experimental result that the signal induced in a compensation coil immediately at the sample surface cannot be balanced by a compensation coil far away. So for correct compensation, one should actually use a compensation coil placed as close to the sample surface as possible. The use of trapezoidal ac fields does, however, allow for an adjustment of the compensation in this region.

Data processing. The compensated signal is then digitalized and stored in a transient recorder (Bryans 514A: 2048 memory locations, $10 \mathrm{bits}$ ). The waveform of the applied field is obtained by measuring the voltage across $R_{\mathrm{m}}=0.1 \Omega$, a resistance in series with the ac coil. This reference signal is recorded simultaneously in a second transient recorder (Datalab DL 905: 1024 memory locations, 8 bits). By short circuiting the input of this transient recorder during the last 64 sampling pulses (switch 1), the dc level of the applied field is also registered. An optimal use of the resolution of the transient recorders is achieved by two small adjustable amplifiers (see Fig. 9, numbers 4 and 5). The sampling process is controlled by the trapezium generator: a trigger pulse at one specific corner of the trapezium is generated to start the process. Also the external clock pulses which regulate the sampling process are provided by this generator. In this way, the sampling process is synchronized with the applied waveform: the number of samples during both the constant and the sloping part of the waveform, respectively, can be adjusted independently. The time interval between two sample pulses is chosen in such a way that during the first period, a sample pulse is generated exactly at each corner of the trapezoidal field. The signalnoise ratio is improved by recording 8 periods of the measuring signal which can be averaged afterwards. During each of these periods, the sample pulse is delayed for $1 / 8$ of the interval between two pulses with respect to the previous period: this provides a better time resolution.

After storing the measuring signal and the reference signal in two transient recorders simultaneously, both signals are recorded successively on magnetic tape with a cassette recorder and fed into the central computer for further analysis. From the reference signal, the amplitude of the applied ac field, the dc level and the value of $\mathrm{d} B_{\mathrm{a}} / \mathrm{d} t$ are derived. The 8 periods of the measuring signal are averaged and subsequently normalized on the response of a normal metal subjected to the same ac field. The response of the normal metal is obtained from the same sample in a small ac field superimposed on a static field well above $B_{\mathrm{c} 2}$. After normalizing, the signal is integrated with respect to time to give the average induction $B_{\mathrm{av}}$ inside the sample. Also the loss during one cycle is calculated. Computer drawings of the various parts of the induced waveform and $B_{\mathrm{av}}$ vs $B_{\text {a }}$ loops can be obtained simply. From these results the critical current density and the flux flow resistivity $\sigma_{\mathrm{ff}}$ can be derived. As a consequence of the synchronization between the applied waveform and the sampling process, the calculations mentioned above are quite simple.

In the case of flux jump experiments this procedure is different since the voltage peak associated with the jump has a duration of a few hundred microseconds which cannot be detected accurately with this method. It is possible, however, to determine the external field at which the jump 
occurs with great accuracy (better than $0.2 \%$ of the amplitude of the applied field, ie better than $0.1 \mathrm{mT}$ ). Also the response during the rest of the period can be detected in the usual way. The voltage peak itself is measured very precisely by sampling the signal with a frequency of $2 \mathrm{MHz}$. A combination of these results now gives a complete description of the response of the superconductors in the form of a $B_{\mathrm{av}}$ vs $B_{\mathrm{a}}$ loop.

\section{The trapezium generator}

Specification. The trapezoidal current through the ac coil is supplied by a voltage controlled, power amplifier. The voltage necessary for such a waveform through a circuit with a resistance $R$ and a self inductance $L$ in series consists of a trapezoidal component and its derivative added in the ratio according to $R$ and $L$ (see Fig. 9). To provide this voltage waveform, a trapezium generator has been designed and built. This generator has to meet the following specifications: one, the ratio between the trapezoidal component and its derivative has to be adjustable; two, the duration of the sloping and the horizontal parts have to be independently variable. For the sloping part a range between $0.5 \mathrm{~ms}$ and $100 \mathrm{~s}$ is required; three, the transient times at the discontinuities have to be less than $10 \mu \mathrm{s}$; four, the drift in the output signal have to be neglectable compared with this signal. By controlling the time scale of this signal with an external pulse generator, it is possible to connect the sampling process with the applied waveform. To achieve this the following additional specifications are required: one, the duration of the sloping part and the horizontal part have to be an integer ( $N 2, N 3$ respectively) times the interval between two sample pulses. $N 2$ and $N 3$ have to be adjustable; two, when an external start pulse is given, the generator must provide a trigger pulse at the beginning of the next rising slope; three, at the beginning of each following period, the sample pulses should be delayed for $1 / 8$ of the interval between two pulses.

In the design described below, these specification have been realized.

Realization. A block diagram of the circuit is given in Fig. 12. The time scale of both the signal and the sampling process is established by means of a digital circuit and controlled by an external pulse generator. Two digital signals are produced which after summation combine to the derivative of a trapezium. The required voltage is now generated by integrating this signal to a trapezium and adding the integrated and non-integrated components. In principle, the frequency of the external clock and the sampling pulses have to be the same. To provide the possibility of an extra delay, a frequency of eight times the desired sample frequency is used. The complete time scale of the signal can be changed simply by varying the frequency of the external clock. The circuit which produces this time scale is shown in Figs 13 and 14. The numbers on the various components refer to standard notation for TTL digital components. The length of the sloping and the constant part of the trapezium, respectively are denoted by $N 2$ and $N 3$ and can be chosen by means of thumbwheels. Division by $N 2$ takes place in the circuit of Fig. 13; a similar circuit used for division by $N 3$ is not given here. After eight pulses at point A (Fig. 14), signal C changes, counter $N 2$ stops and counter $N 3$ is started; after eight $N 3$ pulses, the counting is again switched to counter $N 2$. In this way the input frequency is divided by eight and by $N 2$ and $N 3$ alternately, thereby providing the time

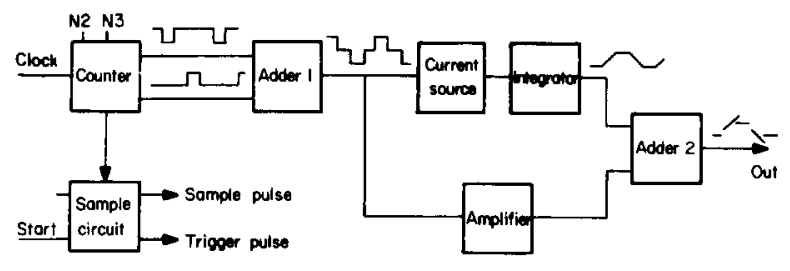

Fig. 12 Block diagram of the trapezium generator. The digital part consists of counter and sampling circuit. In the analogue circuit the two different paths are indicated. The waveform at each stage is also given

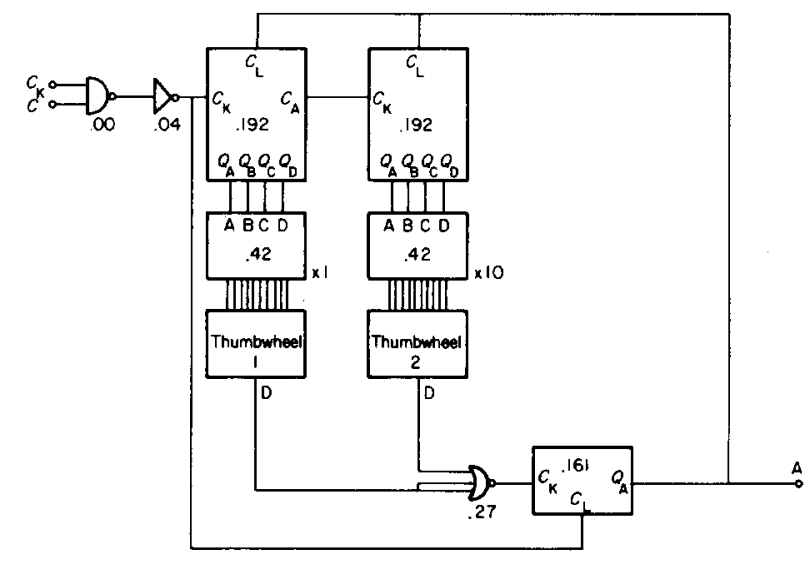

Fig. 13 Digital counter circuit for the number of sample pulses (N2) during the sloping part of the trapezium. N2 can be chosen by means of thumbwheels. At the end of the slpping part signal $C$ is inverted and an identical counter for N3 is established
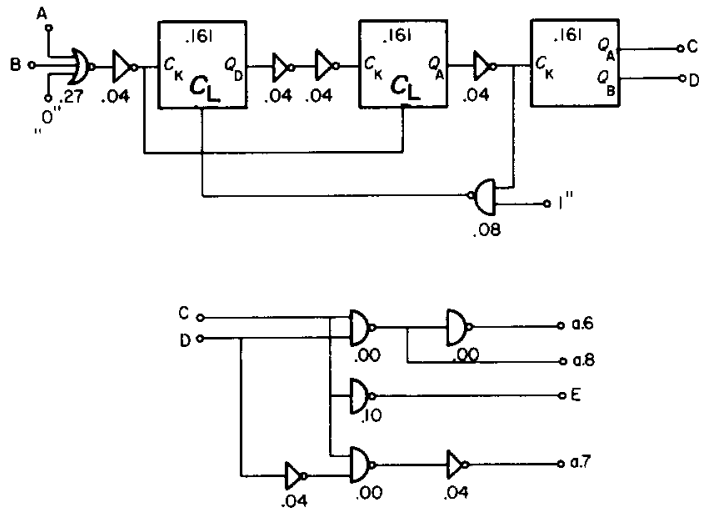

Fig. 14 Coding circuit: the signals $C$ and $D$, derived from the outputs $A$ and $B$ of the counters $N 2$ and $N 3$ are a code for the various intervals of the trapezium

intervals between two successive corners of the trapezium. The signals $C$ and $D$ now can be used as a code for the four different time intervals. From these signals the two components can be derived which, after summation, give the derivative of a trapezium (see Fig. 10).

These signals are also used for the circuit of Fig. 15, which supplies the sample and trigger pulses. The trigger pulse is generated after an external start pulse, which occurs the first time the correct combination of $\mathrm{C}$ and $\mathrm{D}$ arises. This corresponds to the increasing part of the trapezium. The sample pulse is generated by dividing the input frequency by eight. At the beginning of the increasing part of each period however, one single division by nine or ten takes place. In this way the desired delay is realized. The analogue circuit which provides the required voltage waveform is shown in Fig. 16. The input signals are derived from the signals $C$ and D of the digital circuit (see Fig. 14: a7 and a8). With 


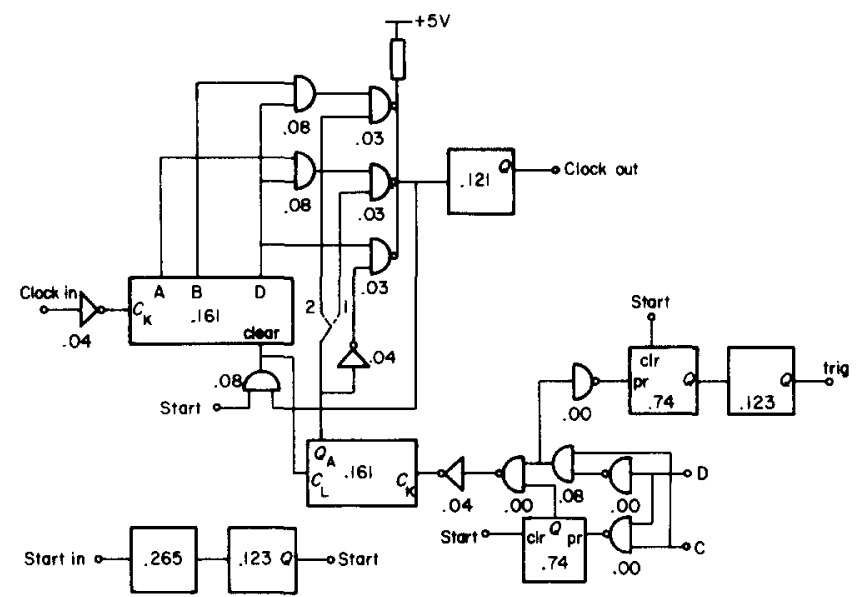

Fig. 15 The sampling circuit provides sample pulses (clock out) and after 'start' a trigger pulse

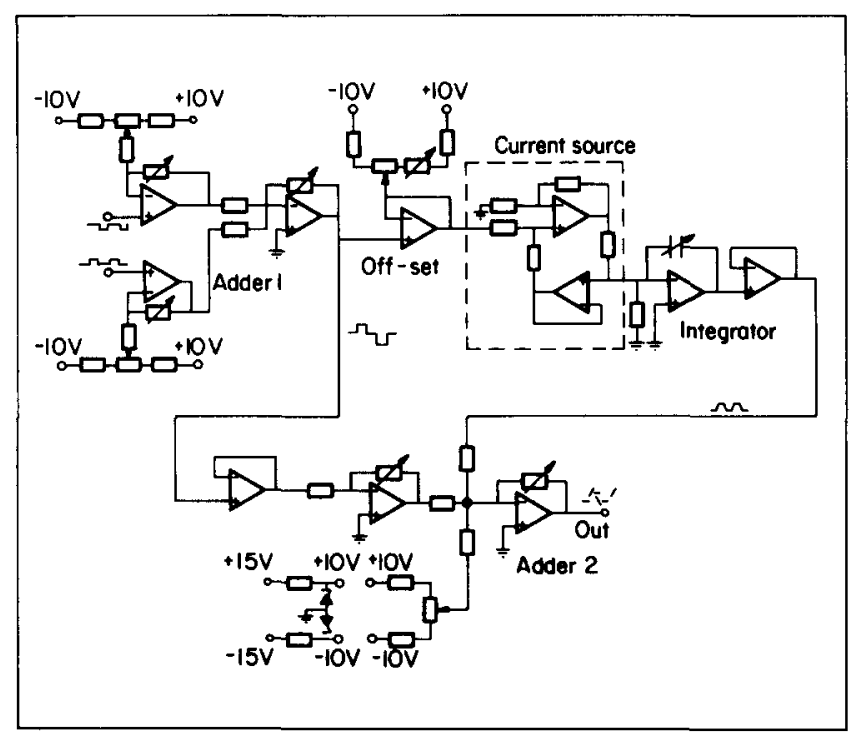

Fig. 16 Analogue circuit. The waveforms at each stage are indicated

a small modification of the digital circuit, the derivative of a double trapezium can also be obtained. These signals can be made equally large by means of two entrance amplifiers; also the dc level can be adjusted in this stage. After summation (adder 1) two different paths are followed, one for each component of the output signal.

The most difficult problem is the integration of this block voltage to a trapezoidal waveform. A small dc voltage at the entrance of the integrator will result in drift at the output. In most commercial triangular generators, this difficulty is removed by reversing $\mathrm{d} U / \mathrm{d} t$ ( $U$ is the output voltage) at a fixed $U$-level. In this way, small variations are intercepted; the result, however, is a variation of the time scale and different slopes during the increasing and decreasing part. For this reason, this method cannot be used here and a very fine dc adjustment is necessary. This has been realized with the help of two ten-turns-potentiometers (offset). The $\pm 10 \mathrm{~V}$ voltages for the dc adjustment are stabilized with the help of zehner diodes. With this arrangement the dc level can be controlled to within $10^{-5} \mathrm{~V}$ while the input voltage during the sloping parts is of the order of $5 \mathrm{~V}$. This means that the drift during one period is a factor of $10^{-5}$ less than the amplitude of the ac field, which is sufficient.

For the integration process itself, a current integrator is used. The input current of this integrator is supplied by a voltage controlled current source with an output current of $2 \mathrm{~mA} \mathrm{~V}^{-1}$ The trapezoidal voltage generated in this way is added to the block signal. The latter can be amplified by a variable factor; in this way the ratio between the two components can be adjusted. The dc level of the output signal can also be adjusted. The required transient time at the discontinuities of less than $10 \mu$ s is realized by a careful selection of electronic components. In all parts of the circuit where discontinuities are involved, fast amplifiers (a HA 2625 wide band operational amplifier with a slew rate of $30 \mathrm{~V} \mathrm{\mu s}^{-1}$ and potentiometers with small self inductances are used. In this way specifications are met easily. A photograph of the generated waveform, together with the resulting current waveform and the signal induced in a pick-up coil, is shown in Fig. 17.

\section{Experimental results and discussion}

In this section the possibilities of the experimental technique described in this paper will be discussed with the help of some results obtained with two polycrystalline slabs of $\mathrm{Nb}$ and $\mathrm{Nb}-50 \% \mathrm{Ti}$.

The behaviour of the Nb-slab can be described by means of reversible surface currents, a bulk critical current density $j_{\mathrm{c}}$ and flux flow. The sample, a Nb-slab $\left(30 \times 5 \times 0.3 \mathrm{~mm}^{3}\right)$ taken from commercial plate, has been heat treated for 10 min in $10^{-6}$ torr just below the melting point. A typical waveform, observed in a small amplitude ac field superimposed on a static field, is shown in Fig. 18 together with the corresponding $B_{\mathrm{av}}-B_{\mathrm{a}}$ curve. The intervals from which the various parameters are determined can be distinguished clearly. A plot of a series of $E$ vs $B_{\mathrm{a}}$ curves for this same sample has already been shown in Fig. 4. Flux distributions and critical current density values have also been :" determined: the results have been published elsewhere..$^{3,33}$ In the case of reversible surface currents, the procedure in the section on separation of loss contributions leads to the reversible magnetization curve. More detailed results will follow in a subsequent paper.

The results obtained on the $\mathrm{Nb}-50 \% \mathrm{Ti}$ slab $(20 \times 5 \times 0.3$ $\mathrm{mm}^{3}$ ) are much easier to interpret since flux flow effects can be neglected. Some flux distributions for various values of $B_{\mathrm{a}}$ obtained with the method described previously are given in Fig. 19; they have already been published elsewhere. ${ }^{34}$ In these profiles three different regions can be distinguished. Firstly, at low external fields surface screening currents in a very narrow layer near the surface can be

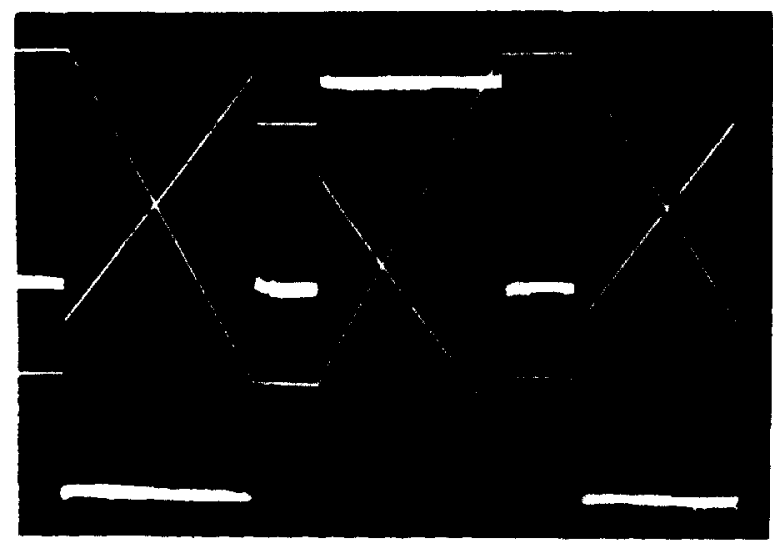

Fig. 17 Output signal of the trapezium generator. Also the resulting trapezoidal waveform of the current and the induced voltage in the compensation coil are shown. This latter waveform is used to control the ratio between the two components of the output signal 

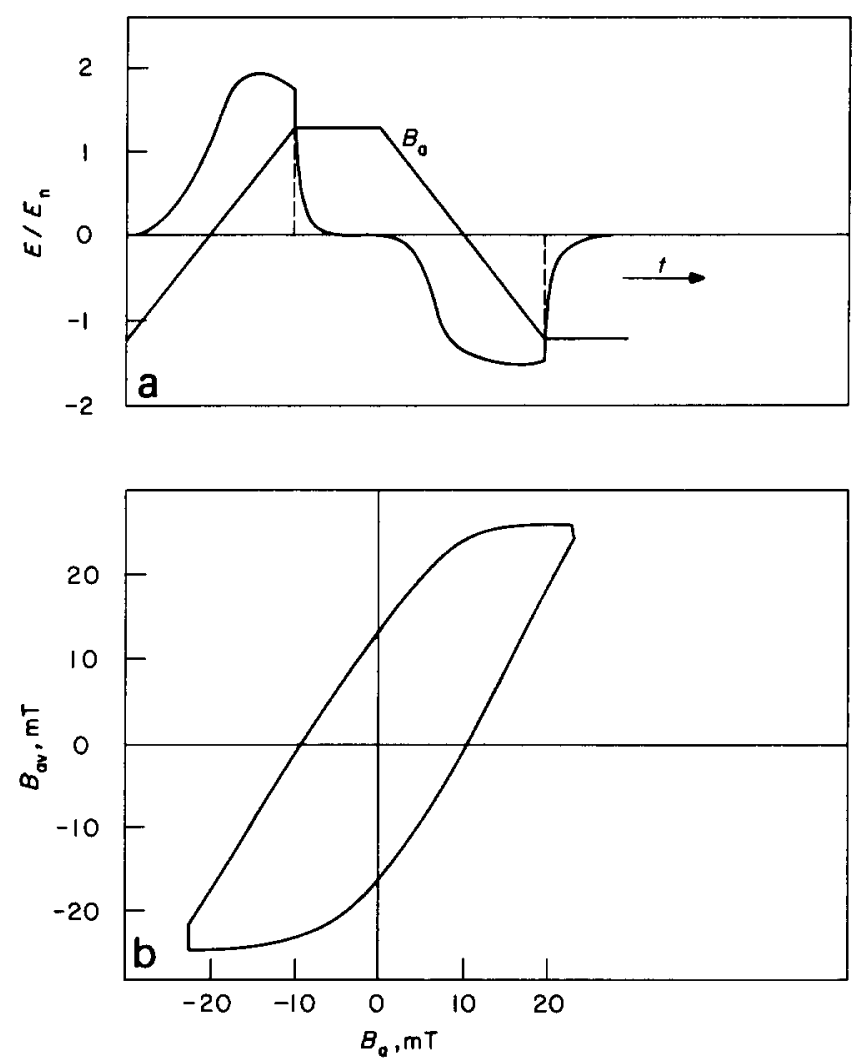

Fig. 18 a - typical $E$ vs $t$ curve for $N b$ in a trapezoidal ac field of small amplitude superposed on a de field $B_{0}\left(B_{0}=137.5 \mathrm{mT}\right.$. $b_{0}=23 \mathrm{mT}, \mathrm{d} B_{\mathrm{a}} / \mathrm{d} t=5.4 \mathrm{~T} \mathrm{~s}^{-1} ; \mathrm{b}$ - corresponding $B_{\mathrm{av}} \mathrm{vs} B_{\mathrm{a}}$ curve

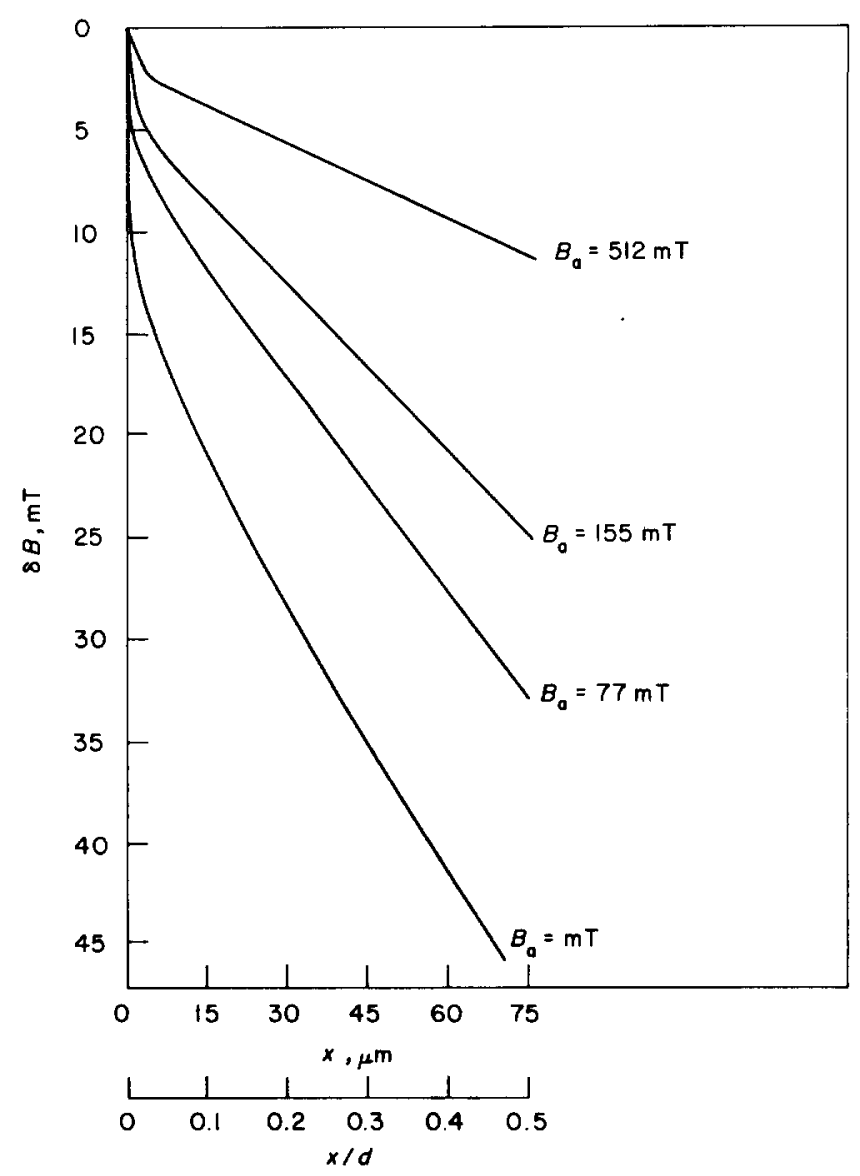

Fig. 19 Flux distribution in $\mathrm{Nb}-50 \% \mathrm{Ti}$ for various values of the external field observed. The thickness of this layer cannot be estimated from the measurements. Secondly, near the surface a small layer with a thickness of about $3 \mu \mathrm{m}$ with a high critical current density $\left(j_{\mathrm{c}} \approx 0.8 \times 10^{5} \mathrm{~A} \mathrm{~cm}^{-2}\right.$ at $\left.B_{\mathrm{a}}=150 \mathrm{mT}\right)$ can be distinguished. Finally, in the bulk of the sample the critical current density is constant. Near the centre plane (not shown in Fig. 19), $j_{\mathrm{c}}$ seems to increase again. This effect is also observed in the $\mathrm{Nb}$ sample and is attributed to an inhomogenous distribution of pinning sites. ${ }^{3}$ The bulk critical current densities have been determined in the field range between 0 and 1.6 T. The results are given in Fig. 20 . Experiments with different amplitude have been used; values from the increasing and decreasing parts, respectively, agree well. An estimate of the error, according to (18) leads to $\epsilon \approx 15 \%$ at $B_{\mathrm{a}}=0.1 \mathrm{~T}$ and $\epsilon<2 \%$ for $B_{\mathrm{a}}>0.5 \mathrm{~T}$. At low fields $\left(B_{\mathrm{a}}<70 \mathrm{mT}\right) j_{\mathrm{c}}$-values cannot be obtained because of the variations of $\partial j_{\mathrm{c}} / \partial B$. The $j_{\mathrm{c}}$-values agree well with results obtained on the same sample with a dc magnetization technique. ${ }^{35}$ The value of $B_{\mathrm{en}}$ and $B_{\text {ex }}$, obtained according to the method presented in the separation of loss contributiond, have already been shown in Fig. 7; in this figure only the curve for a decreasing field has been shown. The distance between these curves at constant average induction $B_{\mathrm{av}}$ as a function of $B_{\mathrm{a}}$, is in agreement with the length of the intervals with induced vol tage $E=0$ immediately after the start of the external sweep. More results on this $\mathrm{Nb}$ $50 \% \mathrm{Ti}$ slab, together with a detailed discussion will be presented elsewhere. ${ }^{25,36}$

In conclusion, it can be said that the use of trapezoidal ac fields in inductive techniques provides a powerful instrument for the study of type II superconductors. This is mainly a consequence of the fact that the sweep rate during the sloping parts of the waveform of the ac fields is constant; apart from this, the presence of time intervals in which there is no variation of applied field is very important. In fact a careful

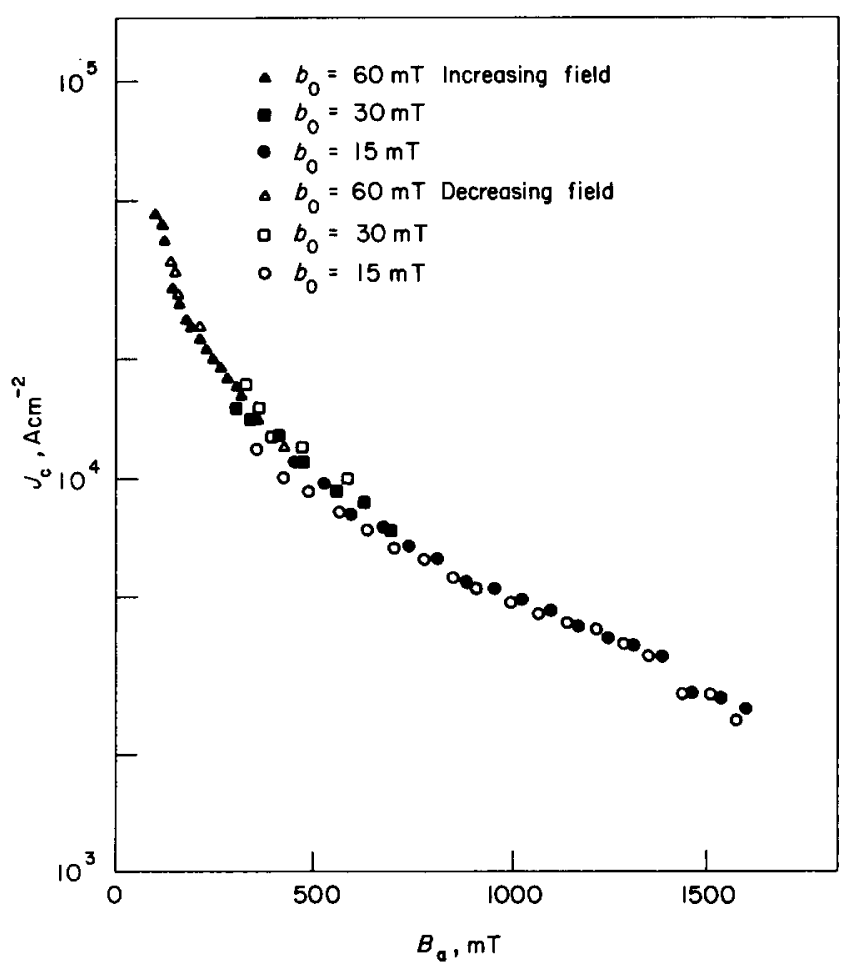

Fig. 20 Critical current values of $\mathrm{Nb}-50 \% \mathrm{Ti}$ as a function of external field. The values have been obtained with ac fields of three different amplitudes and from the increasing as well as decreasing parts of the ac field 
study of the waveform of the induced voltage makes it possible to determine the various parameters of the Critical State Model separately. This may contribute to a better understanding of the losses in type II superconductors under $\mathrm{dc}$ as well as ac conditions.

\section{References}

Bean, C.P. Phys Rev Lett 8 (1962) 250

London, H. Phys Lett 6 (1963) 162

van de Klundert, L.J.M., Gijsbertse, E.A., van de Braak, H.P. Physica 94B (1978) 41

4 Gijsbertse, E.A. thesis, Enschede (1980)

5 Campbell, A.M., Evetts, J.E. Adv Phys 21 (1972) 199

6 Ullmaier, H. Springer Tracts in Modern Physics 76 (1975)

7 van Vijfeycken, A.G. thesis, Amsterdam (1967) Philips Res Repts Suppl 8 (1968)

$8 \quad$ Ullmaier, H. Phys Stat Sol 17 (1966) 631

$9 \quad$ Labusch, R. Crystal Lattice Defects 1 (1969) 1

10 Fietz, W.A., Webb, W.W. Phys Rev 78 (1969) 657

11 Zenneck, J. Ann der Phys IV 9 (1902) 497

12 de Haas, W.J., Du Pré, F.K. Physica 5 (1938) 507

13 Bean, C.P. Rev Mod Phys 36 (1964) 31

14 Campbell, A.M., J Phys C2 (1969) 1492

15 Kroeger, D.M., Koch, C.C., Charlesworth, J.P. J Low Temp Phys 19 (1975) 493

16 Rollins, R.W. Küper, H., Gey, W. J Appl Phys 45 (1974) 5392 van de Klundert, L.J.M., Gijsbertse, E.A., van der Marel, L.C. Proc LT 142 Helsinki (1975) 501

18 Eckert D., Handstein, A. Phys Stat Sol 37A (1976) 171

19 A more precise formulation is that the integral terms in the equations (7) and (11) may be neglected

20 van de Klundert, L.J.M., van de Braak, H.P., Gijsbertse, E.A. Physica 90B (1977) 237

21 Gijsbertse, E.A., van de Klundert, L.J.M. accepted for publication in Physica $B$

22 Walmsley, D.G. J Appl Phys 43 (1972) 615

23 Gijsbertse, E.A., Sikkenga, J., van de Klundert, L.J.M. to be published

24 Kim, Y.B., Hempstead, C.F., Strnad, A.R. Phys Rev 129 (1963) 528

25 Gijsbertse, E.A., de Reuver, J.L., van de Klundert, L.J.M. to be published

26 van de Klundert, L.J.M., Bos, M.R.E., van der Marel, L.C. Cryogenics 17 (1977) 157

Male, J.C. Cryogenics 10 (1970) 381

Easson, R.M., Hlawiczka, P. J Appl Phys 18 (1967) 1237

Penczynski, P. Siemens Forsch Entwickl Ber 2 (1973) 296

Buchhold, T.A. Cryogenics 3 (1963) 141

Melville, P.H. J Phys C4 (1971) 2833

Bussiere, J.F. IEEE Mag 13 (1977) 131

Gijsbertse, E.A., van de Klundert, L.J.M. Phys Lett 77A

(1980) 337

34 Ciszek, M., Kozlowski, G., Tekiel, P., Gijsbertse, E.A., van de Klundert, L.J.M. Phys Lett 77A (1980) 271

35 Ciszek, M., Kozlowski, G. private communications

36 Gijsbertse, E.A., van de Klundert, L.J.M. submitted to Phys Lett

\section{At the heart of the matter}

Without transducers, sophisticated measurement and control would be impossible. They are the heart of the matter, and at Transducer/Tempcon, the annual exhibition devoted exclusively to transducer and temperature control, they are the only thing that matters. Whether you are measuring vibration, velocity, strain, pressure, temperature, displacement flow, noise, torque, level or any other form of energy, it is at Transducer/Tempcon that you will find the transducers that do the trick and their supporting control equipment. Only at this specialist exhibition will you find a full and comprehensive range, covering latest developments in i.c. transducers, ultrasonics etc. on the stands of 130 companies from 10 countries.

for more details;

Trident International Exhibitions Ltd., 21 Plymouth Road, Tavistock, Devon PL19 8AU Tel. (0822) 4671 Telex 45412 TRITAV

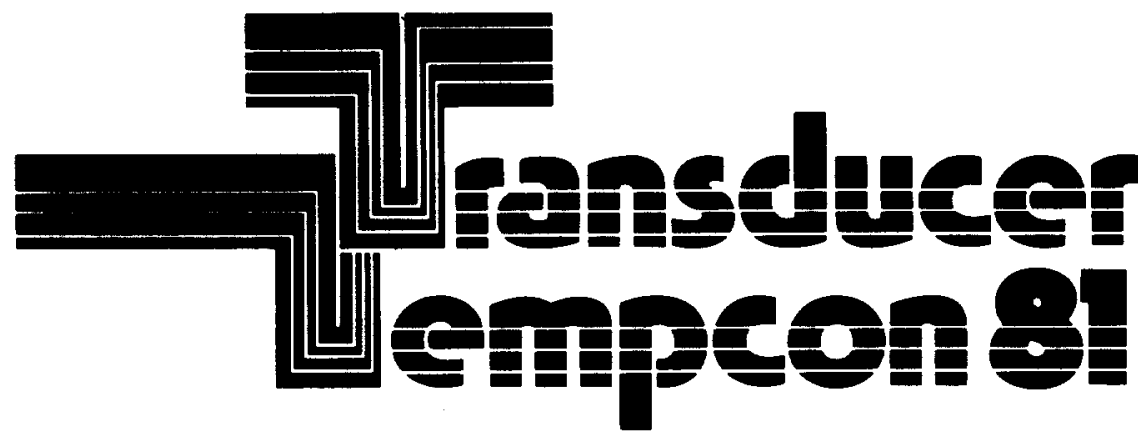

Wembley Conference Centre, London. June 9, 10, 11, 1981 